\title{
Necessary conditions for multiobjective optimal control problems with free end-time*
}

\author{
B. T. Kien† N.-C. Wong ${ }^{\ddagger}$ and J.-C. Yao ${ }^{\S}$
}

January 30, 2008

\begin{abstract}
Necessary conditions of optimality are derived for multiobjective optimal control problems with free end-time, in which the dynamics constraint is modeled as a nonconvex differential inclusion. The obtained results cover some previous results on necessary conditions for multiojective and single objective optimal control problems.
\end{abstract}

Keywords: multiobjective optimal control problems, free end-time, preference, nonconvex differential inclusion, nonsmooth analysis.

\section{Introduction}

The derivation of necessary conditions for multiojective optimal control problems in which the dynamic constraint is modelled as a differential inclusion has been an area research recently. Problems of multiobjective optimal control (MOC for short) naturally arise, for example, in economics (see [6]), in chemical engineering (see [3]) and in multiobjective control design (see [26]). Let us assume that $\prec$ is a preference in $R^{m}$. We are interested in deriving necessary conditions for the problem with free end-times and state constraints

(P) Minimize $g(a, x(a), b, x(b))$

*The authors sincerely thank the referees for their helpful comments and suggestions which improved this manuscript greatly. This research was partially supported by a grant from the National Science Council of Taiwan, R.O.C.

${ }^{\dagger}$ Department of Information and Technology, Hanoi University of Civil Engineering, 55 Giai Phong, Hanoi, Vietnam. Email: kienbt@uce.edu.vn

${ }_{\ddagger}^{\ddagger}$ Department of Applied Mathematics, National Sun Yat-Sen University, Kaohsiung, Taiwan 804. Email: wong@math.nsysu.edu.tw.

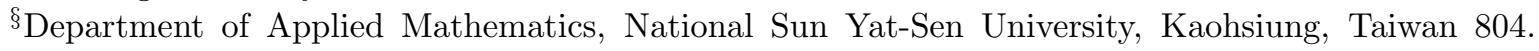
Email: yaojc@math.nsysu.edu.tw. 
over on intervals $[a, b]$ and $\operatorname{arcs} x \in W^{1,1}\left([a, b], R^{n}\right)$ which satisfy

$\dot{x}(t) \in F(t, x(t))$, a.e., $t \in[a, b]$,

$(a, x(a), b, x(b)) \in C$,

where $g: R \times R^{n} \times R \times R^{n} \rightarrow R^{m}$ is a given mapping, $F: R \times R^{n} \rightrightarrows R^{n}$ is a given multifunction, $C$ is a closed set in $R \times R^{n} \times R \times R^{n}$ and $W^{1,1}\left([a, b], R^{n}\right)$ is the space of absolutely continuous functions $x:[a, b] \rightarrow R^{n}$.

Given $x \in W^{1,1}\left([a, b], R^{n}\right)$ we define $x^{e}$ to be an extension of $x$ obtained by constants extension for the left endpoint on $(-\infty, a)$ and from right endpoint on $(b,+\infty)$. A feasible process $([a, b], x)$ comprises a closed interval $[a, b]$ and an arc $x \in W^{1,1}\left([a, b], R^{n}\right)$ which satisfy the constraints of $(\mathrm{P})$. A feasible process $\left(\left[a_{*}, b_{*}\right], x_{*}\right)$ is said to be a local solution of $(\mathrm{P})$ if there do not exist any feasible process $([a, b], x)$ with $d\left(([a, b], x),\left(\left[a_{*}, b_{*}\right], x_{*}\right)\right) \leq \epsilon$ such that $g(a, x(a), b, x(b)) \prec g\left(a_{*}, x_{*}\left(a_{*}\right), b_{*}, x_{*}\left(b_{*}\right)\right)$ for some $\epsilon>0$. Here

$$
d\left(([a, b], x),\left(\left[a^{\prime}, b^{\prime}\right], y\right)\right):=\left|a-a^{\prime}\right|+\left|b-b^{\prime}\right|+\left|x(a)-y\left(a^{\prime}\right)\right|+\int_{a \wedge a^{\prime}}^{b \vee b^{\prime}}\left|\dot{x}^{e}(s)-\dot{y}^{e}(s)\right| d s,
$$

in which $a \wedge a^{\prime}:=\min \left\{a, a^{\prime}\right\}$ and $b \vee b^{\prime}:=\max \left\{b, b^{\prime}\right\}$. We remark that the notion of $W^{1,1}$ local optimizers to differential inclusions was first introduced and studied in [15] under the name of "intermediate local minimizers", which are different from the classical notions of weak and strong local minimizers in variational and optimal control problems.

In the scalar case $(m=1)$, there are several papers dealing with necessary conditions of the Euler-Lagrange type for $(\mathrm{P})$. The generalized Euler-Lagrange condition was first established by Mordukhovich [15] for problems governed by nonconvex, compact-valued, Lipschitzian differential inclusions on the fixed time interval and then was extended to free-time problems in [14]. Further extensions for unbounded differential inclusions were given by Ioffe [8], Loewen and Rockafellar [10], Vinter and Zheng [25] for problems with unbounded differential inclusions on the fixed time interval and then by Vinter and Zheng [23] and Vinter [22] for free-time problems.

Particularly, Vinter [22] provided an efficient scheme for deriving necessary conditions of local optimization solutions of (P) (see [22, Theorem 8.4.1]). A notable feature of the new free end-time necessary conditions is that they cover problems with measurable time dependent data. For such problems, standard analytical techniques for deriving free-time necessary conditions, which depend on a transformation of the time variable, no longer work.

It is natural to ask whether the conclusions of theorems in [22] are still valid for the case of multiobjective optimal control problems. The aim of this paper is to obtain such results for $(\mathrm{P})$.

Unfortunately, the scheme of the proof given by [22] fails to apply to our problem. The reason is that in this case we can not use scalar estimations as well as differentiable property of functions for the problem. However, that scheme helps us derive necessary 
conditions for the Bolza problem with finite Lagrangrian which plays an important role in the establishment of necessary conditions for $(\mathrm{P})$.

In a close connection, recently Zhu [28] had established a result on the Hamiltonian necessary conditions for a nonsmooth multiobjective optimal control problem with endpoint constraints involving regular preferences. This result was extended later by Bellaassali and Jourani [2]. Based on an analysis of Ioffe's scheme [8], as it was mentioned, Bellaassali and Jourani [2] obtained a interesting result on necessary conditions for multiobjective optimal control problems. However, [2] and [28] considered only optimal problems with the fixed time interval.

In order to derive necessary conditions of the Euler-Lagrange type for $(\mathrm{P})$, we use a variant of Ioffe's scheme [8] to reduce the problem to the scalar case as it has been done in [2] and [24]. We then use the Ekeland principle and necessary conditions for the Bolza problem. Together with the maximum theorem and some analytical techniques of nonsmooth analysis we finally obtain desired results.

The rest of the paper contains three sections. In Section 2 we present some notions and auxiliary results involving generalized differentiation. Section 3 is to derive necessary conditions for the Bolza problems. The final section is devoted to deriving necessary conditions for problem $(\mathrm{P})$.

\section{Preliminaries and auxiliary results}

Throughout the paper $B$ stands for the closed unit ball in $R^{n}$ and $R_{\infty}$ stands for $R \cup\{+\infty\}$.

In what follows we often deal with set-valued mappings $\Gamma: R^{n} \rightrightarrows R^{n}$, for which the notation

$$
\operatorname{Limsup}_{x \rightarrow \bar{x}} \Gamma(x):=\left\{x^{*} \in R^{n}: \exists x_{k} \rightarrow \bar{x}, x_{k}^{*} \rightarrow x^{*} \text { with } x_{k}^{*} \in \Gamma\left(x_{k}\right)\right\}
$$

denotes the sequential Painlevé-Kuratowski upper limit of $\Gamma$ at a point $\bar{x} \in R^{n}$. The set

$$
\mathrm{Gph} \Gamma:=\left\{(x, y) \in R^{n} \times R^{n}: y \in \Gamma(x)\right\}
$$

is called the graph of $\Gamma$.

Take a closed set $A \subset R^{n}$ and point $x \in A$. The set

$$
\hat{N}_{A}(x):=\left\{x^{*} \in R^{n}: \limsup _{u \stackrel{A}{\rightarrow} x} \frac{\left\langle x^{*}, u-x\right\rangle}{\|u-x\|} \leq 0\right\}
$$

is called the Fréchet normal cone to $A$ at $x$. Let $\bar{x} \in A$, the set

$$
N_{A}(\bar{x}):=\operatorname{Limsup}_{x \rightarrow \bar{x}} \hat{N}_{A}(x)
$$

is the limiting normal cone to $A$ at $\bar{x}$. 
Given a lower semicontinuos function $f: R^{n} \rightarrow R_{\infty}$ and a point $x \in R^{n}$ such that $f(x)<\infty$, the limiting subdifferential of $f$ at $x$ is the set

$$
\partial f(x)=\left\{x^{*}:\left(x^{*},-1\right) \in N_{\text {epif }}(x, f(x))\right\} .
$$

It is well known that if $f$ is Lipschitz continuous around $x$ with rank $K$, then for any $x^{*} \in \partial f(x)$, one has $\left\|x^{*}\right\| \leq K$. The limiting normal cone and limiting subdifferential were introduced by Mordukhovich [18]. We refer the reader to Chapter 1 in [12] for comprehensive commentaries. Further properties of limiting normal cone and limiting subdifferential can be founded in [12] and [4].

Let $\Gamma: X \subset R^{n} \rightarrow 2^{R^{n}}$ be a multifunction. We now assume that $\Gamma$ has closed values and define the function $\rho_{\Gamma}: X \times R^{n} \rightarrow R$ by

$$
\rho_{\Gamma}(x, y)=d(y, \Gamma(x)):=\inf _{v \in \Gamma(x)}\|y-v\| .
$$

The following property of the subdifferential of $\rho_{F}$ which was first established in [21], will be needed in section 4 .

Lemma 2.1 Assume that $\mathrm{GphF}$ is closed and $(\bar{x}, \bar{y}) \in \mathrm{Gph} \Gamma$. Then one has

$$
N_{\mathrm{Gph} \Gamma}(\bar{x}, \bar{y})=\bigcup_{\lambda \geq 0} \lambda \partial \rho_{\Gamma}(\bar{x}, \bar{y}) .
$$

Moreover, if $\rho_{\Gamma}(x, y)>0$ and $v \in \partial_{y} \rho_{\Gamma}(x, y)$ then there exists a point $z \in \Pi_{\Gamma(x)}(y)$ such that $v=\frac{y-z}{\|y-z\|}$. Here $\Pi_{\Gamma(x)}(y)$ is the set of metric projections of $y$ onto $\Gamma(x)$.

The proof of Lemma 2.1 can be also found in [8], [12] and [24].

Recall that the multifunction $\Gamma: X \subset R^{n} \rightrightarrows R^{n}$ is said to be lower semicontinous (l.s.c.) on $X$ if for each $x_{0} \in X$ and an open set $V$ satisfying $F\left(x_{0}\right) \cap V \neq \emptyset$, there exists a neighborhood $U$ of $x_{0}$ such that $F(x) \cap V \neq \emptyset$ for all $x \in U \cap X$. F is said to be upper semicontinuous (u.s.c.) on $X$ if for each $x_{0} \in X$ and an open set $V$ in $R^{n}$ satisfying $F\left(x_{0}\right) \subset V$, there exists a neighborhood $U$ of $x_{0}$ such that $F(x) \subset V$ for all $x \in U \cap X$. $F$ is said to be continuous on $X$ if it is both l.s.c. and u.s.c. on $X$.

In the sequel we shall need

Lemma 2.2 Let $X \subset R^{n}, Y \subset R^{n}$ be nonempty sets, $\phi: Y \times R^{n} \rightarrow R$ be a continuous function and $\Gamma: X \subset R^{n} \rightrightarrows R^{n}$ be a multifunction with compact values. Assume that $\Gamma$ is Lipschitz continuous on $X$, that is, there exists a constant $k>0$ such that

$$
\Gamma\left(x^{\prime}\right) \subset \Gamma(x)+k\left|x^{\prime}-x\right| B
$$

for all $x, x^{\prime} \in X$. Then the function $M$ defined by

$$
M(x, y)=\max \{\phi(y, u): u \in \Gamma(x)\}
$$

is continuous on $X \times Y$. 
Proof. We first show that $\Gamma$ is l.s.c. on $X$. Indeed, take any point $x_{0} \in X$ and a open set $V$ such that $\Gamma\left(x_{0}\right) \cap V \neq \emptyset$. We want to prove that there exists a neighborhood $U$ of $x_{0}$ such that $\Gamma(x) \cap V \neq \emptyset$ for all $x \in U$. Otherwise, there is a sequence $x_{n} \rightarrow x_{0}$ satisfying $\Gamma\left(x_{n}\right) \cap V=\emptyset$. Take $y_{0} \in \Gamma\left(x_{0}\right) \cap V$. By the property of $\Gamma, d\left(y_{0}, \Gamma\left(x_{n}\right)\right) \leq k\left|x_{0}-x_{n}\right|$. Hence for each $n$, there exists $y_{n} \in \Gamma\left(x_{n}\right)$ such that $\left|y_{0}-y_{n}\right| \leq k\left|x_{0}-x_{n}\right|$. Consequently, $y_{n} \rightarrow y_{0}$ and so $y_{n} \in V$ for $n$ sufficiently large. It follows that $y_{n} \in \Gamma\left(x_{n}\right) \cap V$ for $n$ sufficiently large which is a contradiction. Thus $\Gamma$ is l.s.c. on $X$. By the standard arguments, we can also show that $\Gamma$ is u.s.c. on $X$.

For each $(x, y) \in X \times Y$ we put $z=(x, y)$. Define mappings $\hat{\phi}: R^{n} \times Y \times R^{n} \rightarrow R$ and $\hat{\Gamma}: X \times Y \rightarrow R^{n}$ by $\hat{\phi}(z, u)=\phi(y, u), \hat{\Gamma}(z)=\Gamma(x)$. Then we have

$$
M(x, y)=M(z)=\max \{\hat{\phi}(z, u): u \in \hat{\Gamma}(z)\}
$$

Since $\hat{\Gamma}$ is continuous on $X \times Y$ with compact values and $\hat{\phi}$ is a continuous function, the maximum theorem (see [1, Maximum theorem, p. 116]) implies that $M$ is continuous on $X \times Y$.

We remark that in [13] Mordukhovich and Nam showed that under certain conditions, $M$ is locally Lipschitz continuous (see [13, Theorem 5.2]). However, they required that the cost function $\phi$ is locally Lipschitzian. As we only need the continuity of $M$, in Lemma 2.2 , we did not require that $\phi$ is locally Lipschizian.

The rest of this section is destined for some notion of preferences in $R^{m}$. The concept of a preference first appeared in the value theory of economics. In the area of multiobjective optimization and optimal control much research has been devoted to the weak Pareto solution and its generalizations. The preference relation between vectors $x, y \in R^{m}$ in the sense of weak Pareto is defined by $x \prec y$ if and only if $x_{i} \leq y_{i}$ for $i=1, . ., m$ and at least one of the inequalities is strict. In other words, $x \prec y$ if and only if $x-y \in R_{-}^{m}$ and $x \neq y$, where $R_{-}^{m}:=\left\{z \in R^{m}: z_{i} \leq 0, i=1,2, \ldots, m\right\}$. In this paper we use more general preference relations for which necessary conditions of the weak Pareto solution and its generalization can be derived and refined from our necessary conditions.

Let $\prec$ be a preference in $R^{m}$ and $r \in R^{m}$. We will call the set $\mathcal{L}[r]:=\left\{s \in R^{m}: s \prec r\right\}$ a level set at $r$ and $\overline{\mathcal{L}}[r]$ is the closure of $\mathcal{L}[r]$.

We shall use the following definition (see [12, Dedinition 5.55] and [28]).

Definition 2.3 A preference $\prec$ is closed provided that

(a) for any $r \in R^{n}, r \in \overline{\mathcal{L}}[r]$;

(b) for any $r \prec s, t \in \overline{\mathcal{L}}[r]$ implies that $t \prec s$.

We say that $\prec$ is regular at $\bar{r}$ (in the sense of [28]) provided that

(c)

$$
\operatorname{Limsup}_{r, \theta \rightarrow \bar{r}} N_{\overline{\mathcal{L}}[r]}(\theta) \subset N_{\overline{\mathcal{L}}[\bar{r}]}(\bar{r})
$$


It is noted that the regularity notion for preference was introduced by [17] under the name of normal semicontinuity under which it is studied in Chapter 5 of [12]. In the above definition, the regularity is somewhat different from that in Definition 5.69 of [12], where a preference $\prec$ is regular at $(\bar{\theta}, \bar{r}) \in \mathrm{Gph} \mathcal{L}$ if

$$
\operatorname{Limsup}_{(r, \theta) \stackrel{\operatorname{Gph} \mathcal{L}}{\longrightarrow}(\bar{\theta}, \bar{r})} \hat{N}_{\mathcal{L}[r]}(\theta)=N_{\mathcal{L}[\bar{\theta}]}(\bar{r}) .
$$

Let us give some examples for Definition 2.3.

Example 2.4 (single objective problem). When $m=1$ the relation $r \prec s$ becomes $r<s$. It is obvious that this relation satisfies conditions (a)-(c). Therefore necessary conditions for $(\mathrm{P})$ are true generalizations of necessary conditions for single objective optimal control (see Corollary 4.2).

Example 2.5 (weak Pareto optimal control problem). In a weak Pareto optimal control problem we define the preference by $r \prec s$ iff $r_{i} \leq s_{i}, i=1,2, \ldots, m$, and at least one of the inequalities is strict. It is easy to check that this $\prec$ satisfies (a) and (b) at any $r \in R^{n}$. Moreover, for any $r \in R^{m}, \mathcal{L}[r]=r+R_{-}^{m}$, where $R^{n} m_{-}:=\left\{s \in R^{m}: s_{i} \leq\right.$ $0, i=1,2, \ldots, m\}$. It follows that $N_{\overline{\mathcal{L}}[r]}(\theta) \subset R_{+}^{m}=N_{\overline{\mathcal{L}}[r]}(r)$ for all $r$ and $\theta$. Hence (c) is also satisfied. Thus the necessary conditions for $(\mathrm{P})$ with respect to $\prec$, are true for weak Pareto optimal control problems (see Corollary 4.3).

\section{The Bolza problem with finite Lagrangian}

In this section we derive necessary conditions of the Bolza problem

$$
\begin{aligned}
& \text { Minimize } J(a, b, x):=l(a, x(a), b, x(b))+\int_{a}^{b} L(t, x(t), \dot{x}(t)) d t \\
& \text { over intervals }[a, b] \text { and arcs } x \in W^{1,1}\left([a, b], R^{n}\right),
\end{aligned}
$$

where $l: R \times R^{n} \times R \times R^{n} \rightarrow R_{\infty}$ and $L: R \times R^{n} \times R^{n} \rightarrow R$ are given functions.

A triple $([a, b], x)$ which satisfies the constraint of $(\mathrm{PB})$ is called a feasible process. A feasible process $\left(\left[a_{*}, b_{*}\right], x_{*}\right)$ is a local solution of (BP) if there exists $\epsilon>0$ such that $J(a, b, x) \geq J\left(a_{*}, b_{*}, x_{*}\right)$ for all feasible process satisfying $d\left(([a, b], x),\left(\left[a_{*}, b_{*}\right], x_{*}\right)\right) \leq \epsilon$.

We now fix a feasible process $\left(\left[a_{*}, b_{*}\right], x_{*}\right)$ for the problem and assume the following assumptions which involve positive numbers $\delta, \delta_{0}, \delta_{1}$ :

(BH1) $l$ is Lipschitz continuous near $\left(a_{*}, x_{*}\left(a_{*}\right), b_{*}, x_{*}\left(b_{*}\right)\right)$ with rank $k_{l}$.

(BH2) $L(\cdot, x, \cdot)$ is $\mathcal{L} \times \mathcal{B}$ measurable for each $x \in R^{n}$ and $L(t, \cdot, \cdot)$ is lower semicontinuous for a.e. $t \in\left[a_{*}, b_{*}\right]$.

(BH3) For all $N$ there exists $k_{N} \in L^{1}\left[a_{*}, b_{*}\right]$ such that

$$
\left|L(t, x, v)-L\left(t, x^{\prime}, v\right)\right| \leq k_{N}(t)\left|x-x^{\prime}\right|, L\left(t, x_{*}(t), v\right) \geq-k_{N}(t)
$$


for all $x, x^{\prime} \in x_{*}(t)+\delta B$ and $v \in \dot{x}_{*}(t)+N B$, a.e. $t \in\left[a_{*}, b_{*}\right]$.

(BH4) There exist essentially bounded functions $\bar{u}:\left[a_{*}-\delta_{0}, a_{*}\right] \rightarrow R^{n}$ and $\tilde{u}:\left[b_{*}, b_{*}+\delta_{1}\right] \rightarrow$ $R^{n}$ such that the function $t \mapsto L\left(t, x_{*}\left(a_{*}\right), \bar{u}(t)\right)$ and $t \mapsto L\left(t, x_{*}\left(b_{*}\right), \tilde{u}(t)\right)$ are essentially bounded on $\left[a_{*}-\delta_{0}, a_{*}\right]$ and $\left[b_{*}, b_{*}+\delta_{1}\right]$, respectively. Moreover, there exist positive constants $k_{0}, k_{1}$ such that for all $u \in R^{n}$ one has

$$
\left|L(t, x, u)-L\left(t, x^{\prime}, u\right)\right| \leq k_{0}\left|x-x^{\prime}\right| \forall x, x^{\prime} \in x_{*}\left(a_{*}\right)+\delta B \text {, a.e. } t \in\left[a_{*}-\delta_{0}, a_{*}\right]
$$

and

$$
\left|L(t, x, u)-L\left(t, x^{\prime}, u\right)\right| \leq k_{1}\left|x-x^{\prime}\right| \forall x, x^{\prime} \in x_{*}\left(b_{*}\right)+\delta B \text {, a.e. } t \in\left[b_{*}, b_{*}+\delta_{1}\right] .
$$

Define

$$
\mathcal{H}_{\lambda}(t, x, v, p)=\langle p, v\rangle-\lambda L(t, x, v)
$$

We have the following result on necessary conditions for (BP).

Theorem 3.1 Assume that $\left(\left[a_{*}, b_{*}\right], x_{*}\right)$ is a local minimizer of $(\mathrm{BP})$, for which $J\left(a_{*}, x_{*}, b_{*}\right)<$ $\infty$ and $(\mathrm{BH} 1)-(\mathrm{BH} 3)$ are satisfied.

Then there exist an arc $p \in W^{1,1}\left(\left[a_{*}, b_{*}\right], R^{n}\right)$, real numbers $\xi$, $\eta$ and $\lambda \geq 0$ such that

(i) $\lambda+\|p\|_{\infty}=1$,

(ii) $\dot{p}(t) \in \operatorname{co}\left\{\alpha:(\alpha, p(t)) \in \lambda \partial L\left(t, x_{*}(t), \dot{x}_{*}(t)\right)\right\}$ a.e. $t \in\left[a_{*}, b_{*}\right]$,

(iii) $\left(-\xi, p\left(a_{*}\right), \eta,-p\left(b_{*}\right)\right) \in \lambda \partial l\left(a_{*}, x_{*}\left(a_{*}\right), b_{*}, x_{*}\left(b_{*}\right)\right)$,

(iv) $\left\langle p(t), \dot{x}_{*}(t)\right\rangle-\lambda L\left(t, x_{*}(t), \dot{x}_{*}(t)\right) \geq\langle p(t), v\rangle-\lambda L\left(t, x_{*}(t), v\right)$ for all $v \in R^{n}$, a.e.,

(v)

$$
\xi \leq \lim _{\sigma \rightarrow 0} \operatorname{ess} \sup _{t \in\left[a_{*}-\sigma, a_{*}+\sigma\right]} \mathcal{H}_{\lambda}\left(t, x_{*}(t), \dot{x}_{*}(t), p\left(a_{*}\right)\right)
$$

and

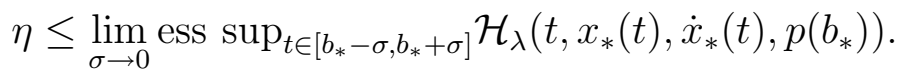

Moreover, if (BH4) holds, then

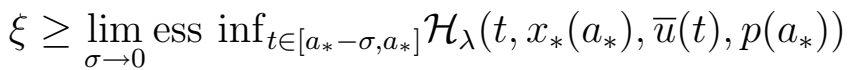

and

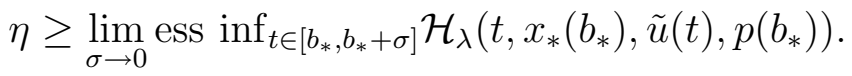

Proof. To prove the theorem, we use a variant of the scheme in [22, Theorem 8.4.1].

Step1. Take $a_{*} \in R, g_{1}: R^{n} \rightarrow R_{\infty}, g_{2}: R \times R^{n} \rightarrow R_{\infty}$ and $g_{3}: R \rightarrow R_{\infty}$. Let $\left(\left[a_{*}, b_{*}\right], x_{*}\right)$ be a $W^{1,1}$ local minimizer for the following problem:

$$
\text { Minimize } g_{1}\left(x\left(a_{*}\right)\right)+g_{2}(b, x(b))+g_{3}(b)+\int_{a_{*}}^{b} L(t, x(t), \dot{x}(t)) d t
$$


over processes $\left(\left[a_{*}, b\right], x\right)$ which satisfy $x \in W^{1,1}\left(\left[a_{*}, b\right]\right)$.

Assume that (BH2) and (BH3) are satisfied, $g_{1}$ is Lipschitz continuous near $x_{*}\left(a_{*}\right), g_{2}$ is twice continuously differentiable near $\left(b_{*}, x_{*}\left(b_{*}\right)\right)$ and $g_{3}$ is Lipschitz continuous near $b_{*}$.

We show that there exist $p \in W^{1,1}$ and $\lambda \geq 0$ such that

(A1) $\lambda+\|p\|_{\infty}=1$

(B1) $\dot{p}(t) \in \operatorname{co}\left\{\alpha:(\alpha, p(t)) \in \lambda \partial L\left(t, x_{*}(t), \dot{x}_{*}(t)\right)\right\}$ a.e.,

(C1) $p\left(a_{*}\right) \in \lambda \partial g_{1}\left(x_{*}\left(a_{*}\right)\right), \quad-p\left(b_{*}\right)=\lambda \nabla_{x} g_{2}\left(b_{*}, x_{*}\left(b_{*}\right)\right)$,

(D1) $\left\langle p(t), \dot{x}_{*}(t)\right\rangle-\lambda L\left(t, x_{*}(t), \dot{x}_{*}(t)\right) \geq\langle p(t), v\rangle-\lambda L\left(t, x_{*}(t), v\right)$ for all $v \in R^{n}$, a.e.,

$$
\lambda \nabla_{b} g_{2}\left(b_{*}, x_{*}\left(b_{*}\right)\right) \leq \operatorname{ess} \sup _{\left[b_{*}-\sigma, b_{*}+\sigma\right]} \mathcal{H}_{\lambda}\left(t, x_{*}(t), \dot{x}_{*}(t), p\left(b_{*}\right)\right)+\lambda k_{3},
$$

in which $k_{3}$ is a Lipschitz constant for $g_{3}$. Moreover, if (BH4) holds then

$$
-\lambda k_{3}+\lim _{\sigma \rightarrow 0} \operatorname{ess} \inf _{\left[b_{*}-\sigma, b_{*}+\sigma\right]} \mathcal{H}_{\lambda}\left(t, x_{*}\left(b_{*}\right), \bar{u}(t), p\left(b_{*}\right)\right) \leq \lambda \nabla_{b} g_{2}\left(b_{*}, x_{*}\left(b_{*}\right)\right) .
$$

Conditions (A1)-(D1) follow directly from the fixed end-time conditions [24, Theorem $3]$. It remains to prove (E1). For $\sigma>0$ sufficiently small, $\left(\left[a_{*}, b_{*}-\sigma\right], x_{*}\right)$ must have cost not less then that of $\left(\left[a_{*}, b_{*}\right], x_{*}\right)$. Hence we have

$g_{2}\left(b_{*}, x_{*}\left(b_{*}\right)\right)+g_{3}\left(b_{*}\right)+\int_{a_{*}}^{b^{*}} L_{*}(t) d t \leq g_{2}\left(b_{*}-\sigma, x_{*}\left(b_{*}-\sigma\right)\right)+g_{3}\left(b_{*}-\sigma\right)+\int_{a_{*}}^{b^{*}-\sigma} L_{*}(t) d t$,

where $\left.L_{*}(t):=L\left(t, x_{*}(t), \dot{x}_{*}(t)\right)\right)$. Since $g_{2}$ is $C^{2}$, we get

$$
0 \leq-\nabla_{b} g_{2}\left(b_{*}, x_{*}\left(b_{*}\right)\right) \sigma-\nabla_{x} g_{2}\left(b_{*}, x_{*}\left(b_{*}\right)\right) \int_{b_{*}-\sigma}^{b^{*}} \dot{x}_{*}(t) d t+o(\sigma)+k_{3} \sigma-\int_{b_{*}-\sigma}^{b^{*}} L_{*}(t) d t .
$$

Consequently,

$$
\begin{aligned}
0 & \leq-\lambda \nabla_{b} g_{2}\left(b_{*}, x_{*}\left(b_{*}\right)\right) \sigma-\lambda \nabla_{x} g_{2}\left(b_{*}, x_{*}\left(b_{*}\right)\right) \int_{b_{*}-\sigma}^{b^{*}} \dot{x}_{*}(t) d t+\lambda o(\sigma)+k_{3} \sigma-\lambda \int_{b_{*}-\sigma}^{b^{*}} L_{*}(t) d t \\
& =-\lambda \nabla_{b} g_{2}\left(b_{*}, x_{*}\left(b_{*}\right)\right) \sigma+\int_{b_{*}-\sigma}^{b^{*}}\left[\left\langle p\left(b_{*}\right), \dot{x}_{*}(t)\right\rangle-\lambda L_{*}(t)\right] d t+\lambda o(\sigma)+\lambda k_{3} \sigma .
\end{aligned}
$$

Hence

$$
\begin{aligned}
\lambda \nabla_{b} g_{2}\left(b_{*}, x_{*}\left(b_{*}\right)\right) & \leq \lim _{\sigma \rightarrow 0} \frac{1}{\sigma} \int_{b_{*}-\sigma}^{b_{*}}\left[\left\langle p\left(b_{*}\right), x_{*}(t)\right\rangle-\lambda L_{*}(t)\right] d t+\lambda k_{3} \\
& \leq \lim _{\sigma \rightarrow 0} \operatorname{ess} \sup _{\left[b_{*}-\sigma, b_{*}+\sigma\right]} \mathcal{H}_{\lambda}\left(t, x_{*}(t), \dot{x}_{*}(t), p\left(b_{*}\right)\right)+\lambda k_{3} .
\end{aligned}
$$

We now assume that (BH4) is fulfilled. Define a multifunction

$$
F:\left[b_{*}, b_{*}+\delta_{1}\right] \times R^{n} \times R \rightarrow R^{n} \times R
$$


by setting $\left.F(t, x, y)=\left\{(u, v) \in R^{n} \times R: v=L(t, x, u)\right)\right\}$. It is clear that for a.e. $t \in\left[b_{*}, b_{*}+\delta_{1}\right]$, the multifunction $(x, y) \mapsto F(t, x, y)$ is Lipschitz continuous with rank $k_{1}$ in a neighborhood of $\left(x_{*}\left(b_{*}\right), y\left(b_{*}\right)\right)$, where $y$ is a given constant function. Define the function $\hat{z}:\left[b_{*}, b_{*}+\delta_{1}\right] \rightarrow R^{n} \times R$ by $\hat{z}(t)=(\hat{x}(t), \hat{y}(t))$, where

$$
\hat{x}(t)=x_{*}\left(b_{*}\right)+\int_{b_{*}}^{t} \tilde{u}(s) d s, \hat{y}(t)=y\left(b_{*}\right)+\int_{b_{*}}^{t} L\left(s, x_{*}\left(b_{*}\right), \tilde{u}(s)\right) d s .
$$

We see that $\dot{\hat{z}}(t) \in F\left(t, x_{*}\left(b_{*}\right), y\left(b_{*}\right)\right)$. For each $\sigma<\delta_{1}$ we put $K_{\sigma}=\exp \left(\int_{b_{*}}^{b_{*}+\sigma} k_{1} d t\right)$, $\rho_{\sigma}(\hat{z})=\int_{b_{*}}^{b_{*}+\sigma} \rho_{F}(t, \hat{z}(t), \dot{\hat{z}}(t)) d t$, where $\rho_{F}(t, z(t), \dot{z}(t)):=d(\dot{z}(t), F(t, z(t))$ and $z(t)=$ $(x(t), y(t))$. Since $t \mapsto \tilde{u}(t)$ and $t \mapsto L\left(t, x_{*}\left(b_{*}\right), \tilde{u}(t)\right)$ are essentially bounded, there exists a constant $M>0$ such that

$$
\left|\hat{z}(t)-\left(x_{*}\left(b_{*}\right), y\left(b_{*}\right)\right)\right| \leq M\left|t-b_{*}\right| .
$$

Hence $\hat{z}(t) \rightarrow\left(x_{*}\left(b_{*}\right), y\left(b_{*}\right)\right)$ as $t \rightarrow b_{*}$. By the Lipschitz continuity of $F$, we have

$$
F\left(t, x_{*}\left(b_{*}\right), y\left(b_{*}\right)\right) \subset F(t, \hat{z}(t))+k_{1}\left|\hat{z}(t)-\left(x_{*}\left(b_{*}\right), y\left(b_{*}\right)\right)\right|
$$

for a.e. $t \in\left[b_{*}, b_{*}+\sigma_{1}\right]$ for some $\sigma_{1}<\delta_{1}$. This implies that $\rho(t, \hat{z}(t), \dot{\hat{z}}(t)) \leq k_{1} M\left|t-b_{*}\right|$ for a.e. $t \in\left[b_{*}, b_{*}+\sigma_{1}\right]$. Hence for all $\sigma \in\left(0, \sigma_{1}\right)$ we have

$$
\rho_{\sigma}(\hat{z})=\int_{b_{*}}^{b_{*}+\sigma} \rho_{F}(t, \hat{z}(t), \dot{\hat{z}}(t)) d t \leq M k_{1} \sigma^{2} .
$$

Consequently, $K_{\sigma} \rho_{\sigma}(\hat{z}) \rightarrow 0$ as $\sigma \rightarrow 0$. By Theorem 3.16 in [5] for each $\sigma \in\left(0, \sigma_{1}\right)$, there exists a solution $z_{\sigma}(t)=\left(x_{\sigma}(t), y_{\sigma}(t)\right), t \in\left[b_{*}, b_{*}+\sigma\right], \dot{z}_{\sigma}(t) \in F\left(t, z_{\sigma}(t)\right)$ with $z_{\sigma}\left(b_{*}\right)=\hat{z}\left(b_{*}\right)$ satisfying

$$
\int_{b_{*}}^{b_{*}+\sigma}\left|\dot{z}_{\sigma}(t)-\dot{\hat{z}}(t)\right| d t \leq K_{\sigma} \int_{b_{*}}^{b_{*}+\sigma} \rho_{F}(t, \hat{z}(t), \dot{\hat{z}}(t)) d t \leq K_{\sigma} M k_{1} \sigma^{2} .
$$

This implies that

$$
\int_{b_{*}}^{b_{*}+\sigma}\left|\dot{x}_{\sigma}(t)-\tilde{u}(t)\right| d t \leq K_{\sigma} M k_{1} \sigma^{2}
$$

and

$$
\int_{b_{*}}^{b_{*}+\sigma} \mid L\left(t, x_{\sigma}(t), \dot{x}_{\sigma}(t)\right)-L\left(t, x_{*}\left(b_{*}\right), \tilde{u}(t) \mid d t \leq K_{\sigma} M k_{1} \sigma^{2} .\right.
$$

Fixing any $\sigma \in\left(0, \sigma_{1}\right)$, we define a function $x$ by consternating $x_{*}(t), a_{*} \leq t \leq b_{*}$ and $x_{\sigma}(t), b_{*} \leq t \leq b_{*}+\sigma$. We therefore obtain a feasible process $\left(\left[a_{*}, b_{*}+\sigma\right], x\right)$. Since $\left(\left[a_{*}, b_{*}+\sigma\right], x\right)$ must have cost not less then that of $\left(\left[a_{*}, b_{*}\right], x_{*}\right)$ we conclude that

$g_{2}\left(b_{*}, x_{*}\left(b_{*}\right)\right)+g_{3}\left(b_{*}\right)+\int_{a_{*}}^{b^{*}} L_{*}(t) d t \leq g_{2}\left(b_{*}+\sigma, x\left(b_{*}+\sigma\right)\right)+g_{3}\left(b_{*}+\sigma\right)+\int_{a_{*}}^{b^{*}+\sigma} L(t, x(t), \dot{x}(t)) d t$. 
Hence

$$
\begin{aligned}
0 & \leq \nabla_{b} g_{2}\left(b_{*}, x_{*}\left(b_{*}\right)\right) \sigma+o(\sigma)+k_{3} \sigma+\int_{b_{*}}^{b_{*}+\sigma} \nabla_{x} g_{2}\left(b_{*}, x_{*}\left(b_{*}\right)\right) \dot{x}_{\sigma}(t) d t+\int_{b_{*}}^{b_{*}+\sigma} L\left(t, x_{\sigma}(t), \dot{x}_{\sigma}(t)\right) d t \\
& \leq \nabla_{b} g_{2}\left(b_{*}, x_{*}\left(b_{*}\right)\right) \sigma+o(\sigma)+k_{3} \sigma+\int_{b_{*}}^{b_{*}+\sigma} \nabla_{x}\left\langle g_{2}\left(b_{*}, x_{*}\left(b_{*}\right)\right), \tilde{u}(t)\right\rangle d t+ \\
& +\left|\nabla_{x} g_{2}\left(b_{*}, x_{*}\left(b_{*}\right)\right)\right| K_{\sigma} M k_{1} \sigma^{2}+\int_{b_{*}}^{b_{*}+\sigma} L\left(t, x_{*}\left(b_{*}\right), \dot{\tilde{u}}(t)\right) d t+K_{\sigma} M k_{1} \sigma^{2} .
\end{aligned}
$$

Multiplying the latter inequality by $\lambda \geq 0$ and dividing by $\sigma>0$ yields

$$
\begin{aligned}
& -\lambda k_{3}+\frac{1}{\sigma} \int_{b_{*}}^{b_{*}+\sigma}\left[p\left(b_{*}\right) \tilde{u}(t)-\lambda L\left(t, x_{*}\left(b_{*}\right), \tilde{u}(t)\right)\right] d t \leq \\
& \leq K_{\sigma} M k_{1} \sigma\left(\nabla_{x} g_{2}\left(b_{*}, x_{*}\left(b_{*}\right)\right)+1\right)+\lambda \nabla_{b} g_{2}\left(b_{*}, x_{*}\left(b_{*}\right)\right) .
\end{aligned}
$$

This implies that

$$
-\lambda k_{3}+\lim _{\sigma \rightarrow 0} \operatorname{ess} \inf _{\left[b_{*}-\sigma, b_{*}+\sigma\right]} \mathcal{H}_{\lambda}\left(t, x_{*}\left(b_{*}\right), \tilde{u}(t), p\left(b_{*}\right)\right) \leq \lambda \nabla_{b} g_{2}\left(b_{*}, x_{*}\left(b_{*}\right)\right) .
$$

Thus assertions of Step 1 are obtained.

Step 2. Take $a_{*} \in R$ and $g: R \times R^{n} \times R^{n} \rightarrow R_{\infty}$. Let $\left(\left[a_{*}, b_{*}\right], x_{*}\right)$ be a $W^{1,1}$ local minimizer for the following problem:

Minimize $g\left(x\left(a_{*}\right), b, x(b)\right)+\int_{a_{*}}^{b} L(t, x(t), \dot{x}(t)) d t$

over processes $\left(\left[a_{*}, b\right], x\right)$ which satisfy $x \in W^{1,1}\left(\left[a_{*}, b\right]\right)$.

Assume that (BH2)-(BH3) are satisfied and $g$ is Lipschitz continuous near $\left(x_{*}\left(a_{*}\right), b_{*}, x_{*}\left(b_{*}\right)\right)$ with a rank $k_{g}$. We show that there exist $p \in W^{1,1}$, real numbers $\eta$ and $\lambda \geq 0$ such that

(A2) $\lambda+\|p\|_{\infty}+|\eta|=1$,

(B2) $\dot{p}(t) \in \operatorname{co}\left\{\alpha:(\alpha, p(t)) \in \lambda \partial L\left(t, x_{*}(t), \dot{x}_{*}(t)\right)\right\}$ a.e.,

(C2) $\left(p\left(a_{*}\right), \eta,-p\left(b_{*}\right)\right) \in \lambda \partial g\left(x_{*}\left(a_{*}\right), b_{*}, x_{*}\left(b_{*}\right)\right)$,

(D2) $\left\langle p(t), \dot{x}_{*}(t)\right\rangle-\lambda L\left(t, x_{*}(t), \dot{x}_{*}(t)\right) \geq\langle p(t), v\rangle-\lambda L\left(t, x_{*}(t), v\right)$ for all $v \in R^{n}$, a.e.,

$$
\eta \leq \lim _{\sigma \rightarrow 0} \operatorname{ess} \sup _{\left[b_{*}-\sigma, b_{*}\right]} \mathcal{H}_{\lambda}\left(t, x_{*}(t), \dot{x}_{*}(t), p\left(b_{*}\right)\right) .
$$

Moreover if (BH4) holds, then

$$
\eta \geq \lim _{\sigma \rightarrow 0} \operatorname{ess} \inf _{\left[b_{*}, b_{*}+\sigma\right]} \mathcal{H}_{\lambda}\left(t, x_{*}\left(b_{*}\right), \tilde{u}(t), p\left(b_{*}\right)\right) .
$$

Take a sequence $K_{i} \rightarrow \infty$ and define

$$
J_{i}(b, x, \tau, y):=g\left(x\left(a_{*}\right), \tau\left(a_{*}\right), y\left(a_{*}\right)\right)+\int_{a_{*}}^{b} L(t, x(t), \dot{x}(t)) d t+K_{i}\left(|\tau(b)-b|^{2}+|y(b)-x(b)|^{2}\right),
$$


where $\tau$ and $y$ are constant functions. Denote by $W$ the set of all $\left(\left[a_{*}, b\right], z=(x, \tau, y)\right)$ such that $x \in W^{1,1}\left(\left[a_{*}, b\right], R^{n}\right), \tau \in R, y \in R^{n}$. With respect to the metric

$d\left(\left(\left[a_{*}, b\right],(x, \tau, y)\right),\left(\left[a_{*}, b^{\prime}\right],\left(x^{\prime}, \tau^{\prime}, y^{\prime}\right)\right)=\left|b-b^{\prime}\right|+\left|x\left(a_{*}\right)-x^{\prime}\left(a_{*}\right)\right|+\left\|\dot{x}^{e}-\dot{x}^{\prime e}\right\|+\left|\tau-\tau^{\prime}\right|+\left|y-y^{\prime}\right|\right.$,

$W$ is complete and $J_{i}$ is continuous. Let us define a sequence $\epsilon_{i}$ by

$$
\epsilon_{i}^{2}:=J_{i}\left(b_{*}, x_{*}, b_{*}, x_{*}\left(b_{*}\right)\right)-\inf _{W} J_{i}(b, x, \tau, y) .
$$

By similar arguments as in Step 5, we can show that $\epsilon_{i} \rightarrow 0$. The Ekeland Principle now gives us, for each $i$, a point $\left(b_{i}, x_{i}, \tau_{i}, y_{i}\right)$ in $W$ such that

$$
\begin{gathered}
d\left[\left(b_{i}, x_{i}, \tau_{i}, y_{i}\right),\left(b_{*}, x_{*}, b_{*}, x_{*}\left(b_{*}\right)\right)\right] \leq \epsilon_{i} \\
J_{i}\left(b_{i}, x_{i}, \tau_{i}, y_{i}\right) \leq J_{i}(b, x, \tau, y)+\epsilon_{i} d\left[\left(b_{i}, x_{i}, \tau_{i}, y_{i}\right),(b, x, \tau, y)\right] \forall(b, x, \tau, y) \in W .
\end{gathered}
$$

From (1), it follows that $b_{i} \rightarrow b_{*}, \tau_{i} \rightarrow b_{*}, y_{i} \rightarrow x_{*}\left(b_{*}\right), x_{i}^{e} \rightarrow x_{*}^{e}$ uniformly, $\dot{x}_{i}^{e} \rightarrow \dot{x}_{*}^{e}$ a.e. and in $L^{1}$. Also, $(2)$ implies that $\left(b_{i}, \tau_{i}, x_{i}, y_{i}\right)$ is a $\mathrm{W}$ minimizer of the functional

$$
\begin{aligned}
& \tilde{J}_{i}(b, z):=g\left(x\left(a_{*}\right), \tau\left(a_{*}\right), y\left(a_{*}\right)\right)+\epsilon_{i}\left(\left|\tau\left(a_{*}\right)-\tau_{i}\right|+\left|x\left(a_{*}\right)-x_{i}\left(a_{*}\right)\right|+\left|y\left(a_{*}\right)-y_{i}\right|\right)+ \\
& +K_{i}\left(|\tau(b)-b|^{2}+|y(b)-x(b)|^{2}\right)+\epsilon_{i}\left(\left|b-b_{i}\right|+\int_{b}^{b \vee b_{i}}\left|\dot{x}_{i}^{e}(t)\right| d t\right)+ \\
& +\int_{a_{*}}^{b}\left(L(t, x(t), \dot{x}(t))+\epsilon_{i}\left|\dot{x}-\dot{x}_{i}^{e}\right|\right) d t
\end{aligned}
$$

Put

$$
\begin{gathered}
g_{1}\left(z\left(a_{*}\right)\right)=g\left(x\left(a_{*}\right), \tau\left(a_{*}\right), y\left(a_{*}\right)\right)+\epsilon_{i}\left(\left|\tau\left(a_{*}\right)-\tau_{i}\right|+\left|x\left(a_{*}\right)-x_{i}\left(a_{*}\right)\right|+\left|y\left(a_{*}\right)-y_{i}\right|\right), \\
g_{2}(b, z(b))=K_{i}\left(|\tau(b)-b|^{2}+|y(b)-x(b)|^{2}\right)
\end{gathered}
$$

and

$$
g_{3}(b)=\epsilon_{i}\left(\left|b-b_{i}\right|+\int_{b}^{b \vee b_{i}}\left|\dot{x}_{i}^{e}(t)\right| d t\right) .
$$

According to Step 1, there exist $p_{i}$, real numbers $\lambda_{i} \geq 0, \eta_{i}$ and $r_{i}$ such that

(i) $\lambda_{i}+\left|\eta_{i}\right|+\left|r_{i}\right|+\left\|p_{i}\right\|_{\infty}=1$

(ii) $\dot{p}_{i}(t) \in \operatorname{co}\left\{\alpha:\left(\alpha, p_{i}(t)\right) \in \lambda_{i} \partial L\left(t, x_{i}(t), \dot{x}_{i}(t)\right)+\epsilon_{i} \lambda_{i}\{0\} \times B\right\}$ a.e. $t \in\left[a_{*}, b_{i}\right]$,

(iii) $\left(p_{i}\left(a_{*}\right), \eta_{i}, r_{i}\right) \in \lambda_{i} \partial g\left(x_{i}\left(a_{*}\right), \tau_{i}, y_{i}\right)+\lambda_{i} \epsilon_{i} B \times B \times B$ and

$-\left(p_{i}\left(b_{i}\right), \eta_{i}, r_{i}\right)=\lambda_{i} \nabla_{z} g_{2}\left(b_{i}, x_{i}\left(b_{i}\right), \tau_{i}\left(b_{i}\right), y_{i}\left(b_{i}\right)\right)$,

(iv) $\left\langle p_{i}(t), \dot{x}_{i}(t)\right\rangle-\lambda_{i} L\left(t, x_{i}(t), \dot{x}_{i}(t)\right) \geq\left\langle p_{i}(t), v\right\rangle-\lambda L\left(t, x_{i}(t), v\right)-\lambda_{i} \epsilon_{i}\left|v-\dot{x}_{i}\right|$ for all $v \in R^{n}$ and a.e. $t \in\left[a_{*}, b_{i}\right]$.

( v)

$$
\lambda_{i} \nabla_{b} g_{2}\left(b_{i}, x_{i}\left(b_{i}\right), \tau_{i}\left(b_{i}\right), y_{i}\left(b_{i}\right)\right) \leq \lim _{\sigma \rightarrow 0} \operatorname{ess} \sup _{\left[b_{i}-\sigma, b_{i}\right]} \mathcal{H}_{\lambda_{i}}\left(t, x_{i}(t), \dot{x}_{i}(t), p_{i}\left(b_{i}\right)\right)+\lambda_{i} \epsilon_{i} k_{3} .
$$


Assume that (BH4) is fulfilled. Putting $\tilde{u}_{i}=\tilde{u}^{e}$, we see that functions $u_{i}$ and

$$
t \mapsto L\left(t, x_{i}\left(b_{i}\right), u_{i}(t)\right)+\epsilon_{i}\left|\tilde{u}_{i}(t)\right|
$$

are essentially bounded on $\left[b_{i}, b_{i}+\delta_{1}\right]$. Moreover for $i$ sufficiently large, the function

$$
x \mapsto L(t, x, u)+\epsilon_{i}\left|u-x_{i}^{e}(t)\right|
$$

is Lipschitz continuous with rank $k_{1}$ for a.e. $t \in\left[b_{i}, b_{i}+\delta_{1}\right]$.

By the conclusion of Step 1, one has

$$
\begin{aligned}
& \lambda_{i} \nabla_{b} g_{2}\left(b_{i}, x_{i}\left(b_{i}\right), \tau_{i}\left(b_{i}\right), y_{i}\left(b_{i}\right)\right) \geq \\
& \geq-\lambda_{i} \epsilon_{i} k_{3}+\lim _{\sigma \rightarrow 0} \operatorname{ess} \inf _{\left[b_{i}, b_{i}+\sigma\right]}\left[\left\langle p_{i}\left(b_{i}\right), u_{i}(t)\right\rangle-\lambda_{i} L\left(t, x_{i}\left(b_{i}\right), \tilde{u}_{i}(t)\right)-\lambda_{i} \epsilon_{i}\left|\tilde{u}_{i}(t)\right|\right] .
\end{aligned}
$$

From (iii) we have $-p_{i}\left(b_{i}\right)=-2 \lambda_{i} K_{i}\left(y_{i}\left(b_{i}\right)-x_{i}\left(b_{i}\right)\right),-\eta_{i}=2 \lambda_{i} K_{i}\left(\tau_{i}-b_{i}\right),-r_{i}=$ $2 \lambda_{i} K_{i}\left(y_{i}\left(b_{i}\right)-x_{i}\left(b_{i}\right)\right)$. Hence $-p_{i}\left(b_{i}\right)=r_{i}$ and $\lambda_{i} \nabla_{b} g_{2}\left(b_{i}, x_{i}\left(b_{i}\right), \tau_{i}\left(b_{i}\right), y_{i}\left(b_{i}\right)\right)=\eta_{i}$.

Since $p_{i}^{\prime} s$ are bounded and their derivatives are bounded by an integrable function, $p_{i} \rightarrow p$ uniformly and $\dot{p}_{i} \rightarrow \dot{p}$ weakly in $L^{1}$ for some $p \in W^{1,1}$. A further subsequence extraction ensures that $\lambda_{i} \rightarrow \lambda, \eta_{i} \rightarrow \eta$ for some $\lambda \geq 0$ and $\eta$. By passing to the limits as $i \rightarrow \infty$ in (i)-(v), we obtain (B2)-(E2).

Since $\lambda_{i}+\left\|p_{i}\right\|_{\infty}+\left|\eta_{i}\right| \neq 0$, by scaling multipliers we can arrange so that $\lambda_{i}+\left\|p_{i}\right\|_{\infty}+$ $\left|\eta_{i}\right|=1$. Letting $i \rightarrow \infty$ we obtain (A2). The proof of Step 2 is complete.

Step 3. (Necessary conditions for fixed right end-time problem). Take $b_{*} \in R$ and $g$ : $R \times R^{n} \times R^{n} \rightarrow R_{\infty}$. Let $\left(\left[a_{*}, b_{*}\right], x_{*}\right)$ be a $W^{1,1}$ local solution of the problem:

Minimize $g\left(a, x(a), x\left(b_{*}\right)\right)+\int_{a}^{b_{*}} L(t, x(t), \dot{x}(t)) d t$

over processes $\left(\left[a, b_{*}\right], x\right)$ which satisfy $x \in W^{1,1}\left(\left[a, b_{*}\right], R^{n}\right)$.

Assume that (BH2),(BH3) are satisfied and $g$ is Lipschitz continuous in a neighborhood of $\left(a_{*}, x_{*}\left(a_{*}\right), x *\left(b_{*}\right)\right)$. We show that there exist $p$, real numbers $\xi$ and $\lambda \geq 0$ such that

(A3) $\lambda+\|p\|_{\infty}+|\xi|=1$

(B3) $\dot{p}(t) \in \operatorname{co}\left\{\alpha:(\alpha, \mathrm{p}(\mathrm{t})) \in \lambda \partial \mathrm{L}\left(\mathrm{t}, \mathrm{x}_{*}(\mathrm{t}), \dot{\mathrm{x}}_{*}(\mathrm{~s})\right)\right\}$ a.e. $t \in\left[a_{*}, b_{*}\right]$,

(C3) $\left(-\xi, p\left(a_{*}\right),-p\left(b_{*}\right)\right) \in \lambda \partial g\left(a_{*}, x_{*}\left(a_{*}\right), x_{*}\left(b_{*}\right)\right)$,

(D3) $\left\langle p(t), \dot{x}_{*}(t)\right\rangle-\lambda L\left(t, x_{*}(t), \dot{x}_{*}(t)\right) \geq\langle p(t), v\rangle-\lambda L\left(t, x_{*}(t), v\right)$ for all $v \in R^{n}$, a.e. $t \in\left[a_{*}, b_{*}\right]$,

(E3)

$$
\xi \leq \lim _{\sigma \rightarrow 0} \operatorname{ess} \sup _{\left[a_{*}, a_{*}+\sigma\right]} \mathcal{H}_{\lambda}\left(t, x_{*}(t), \dot{x}_{*}(t), p\left(a_{*}\right)\right)
$$

Moreover,

$$
\xi \geq \lim _{\sigma \rightarrow 0} \operatorname{ess} \inf _{\left[a_{*}-\sigma, a_{*}\right]} \mathcal{H}_{\lambda}\left(t, x_{*}\left(a_{*}\right), \bar{u}(t), p\left(a_{*}\right)\right)
$$

whenever (BH4) is fulfilled. 
Put $a_{*}^{\prime}=-b_{*}, b^{\prime}=-a, b_{*}^{\prime}=-a_{*}, x^{\prime}(s)=x(-s), \bar{u}^{\prime}(s)=-\bar{u}(-s), x_{*}^{\prime}(s)=x_{*}(-s)$, $g^{\prime}(t, x, y)=g(-t, x, y)$ and $L^{\prime}(s, x, y)=L(-s, x,-y)$. By considering a change of independent variable $s=-t$, it follows that $\left(\left[a_{*}^{\prime}, b_{*}^{\prime}\right], x_{*}^{\prime}\right)$ is a solution of the problem

Minimize $g^{\prime}\left(b^{\prime}, x\left(b^{\prime}\right), x\left(a_{*}^{\prime}\right)\right)+\int_{a_{*}^{\prime}}^{b^{\prime}} L^{\prime}\left(s, x^{\prime}(s), \dot{x}^{\prime}(s)\right) d t$ over processes $\left(\left[a_{*}^{\prime}, b^{\prime}\right], x^{\prime}\right)$ which satisfy $x^{\prime} \in W^{1,1}\left(\left[a_{*}^{\prime}, b^{\prime}\right], R^{n}\right)$.

According to Step 2, there exist $p^{\prime}, \mu^{\prime}, \gamma^{\prime}, \lambda^{\prime}$ and $\eta^{\prime}$ such that

(i) $\lambda^{\prime}+\left\|p^{\prime}\right\|_{\infty}+\left|\eta^{\prime}\right|=1$,

(ii) $\dot{p}^{\prime}(s) \in \operatorname{co}\left\{\alpha:\left(\alpha, \mathrm{p}^{\prime}(\mathrm{s})\right) \in \lambda^{\prime} \partial \mathrm{L}^{\prime}\left(\mathrm{s}, \mathrm{x}_{*}^{\prime}(\mathrm{s}), \dot{\mathrm{x}}_{*}^{\prime}(\mathrm{s})\right)\right\}$ a.e. $s \in\left[a^{\prime}, b_{*}^{\prime}\right]$,

(iii) $\left(\eta^{\prime},-p^{\prime}\left(b_{*}^{\prime}\right), p^{\prime}\left(a_{*}^{\prime}\right)\right) \in \lambda^{\prime} \partial g^{\prime}\left(b_{*}^{\prime}, x_{*}^{\prime}\left(b_{*}^{\prime}\right), x_{*}^{\prime}\left(a_{*}\right)\right)$,

(iv) $\left\langle p^{\prime}(s), \dot{x}_{*}^{\prime}(s)\right\rangle-\lambda^{\prime} L^{\prime}\left(s, x_{*}(s), \dot{x}_{*}^{\prime}(s)\right) \geq\left\langle p^{\prime}(s), v\right\rangle-\lambda^{\prime} L^{\prime}\left(s, x_{*}^{\prime}(s), v\right)$ for all $v \in R^{n}$, a.e., ( $\mathrm{v})$

$$
\eta^{\prime} \leq \lim _{\sigma \rightarrow 0} \operatorname{ess} \sup _{\left[b_{*}^{\prime}-\sigma, b_{*}^{\prime}\right]}\left[\left\langle p^{\prime}\left(b_{*}^{\prime}\right), \dot{x}_{*}^{\prime}(s)\right\rangle-\lambda L^{\prime}\left(s, x_{*}^{\prime}(s), \dot{x}_{*}^{\prime}(s)\right)\right]
$$

Moreover,

$$
\left.\eta^{\prime} \geq \lim _{\sigma \rightarrow 0} \operatorname{ess} \inf _{\left[b_{*}^{\prime}, b_{*}^{\prime}+\sigma\right]}\left[\left\langle p^{\prime}\left(b_{*}^{\prime}\right), \bar{u}^{\prime}(s)\right\rangle-\lambda L^{\prime}\left(s, x_{*}^{\prime}(s), \bar{u}^{\prime}(s)\right)\right)\right]
$$

whenever (BH6) is fulfilled.

Put $\xi=\eta^{\prime}, \lambda=\lambda^{\prime}$ and $p(s)=-p^{\prime}(-s)$. By simple computation we obtain (A3)-(E3) from assertions (i)-(v).

Step 4. Take $g_{1}, g_{2}: R \times R^{n} \rightarrow R_{\infty}, g_{3}: R \rightarrow R_{\infty}$. Let $\left(\left[a_{*}, b_{*}\right], x_{*}\right)$ be a solution of the problem:

Minimize $g_{1}(a, x(a))+g_{2}(b, x(b))+g_{3}(b)+\int_{a}^{b} L(t, x(t), \dot{x}(t)) d t$ over processes $([a, b], x)$ which satisfy $x \in W^{1,1}\left([a, b], R^{n}\right)$.

Assume that (BH2) and (BH3) are satisfied, $g_{1}$ is Lipschitz continuous near $\left(a_{*}, x_{*}\left(a_{*}\right)\right)$, $g_{2}$ is twice differentiable near $\left(b_{*}, x_{*}\left(b_{*}\right)\right)$ and $g_{3}$ is Lipschitz continuous near $b_{*}$ with rank $k_{3}$.

Fixing $b=b_{*}$, we see that $\left(\left[a_{*}, b_{*}\right], x_{*}\right)$ is a solution of the problem

Minimize $g_{1}(a, x(a))+g_{2}\left(b_{*}, x\left(b_{*}\right)\right)+g_{3}\left(b_{*}\right)+\int_{a}^{b_{*}} L(t, x(t), \dot{x}(t)) d t$ over processes $\left(\left[a, b_{*}\right], x\right)$ which satisfy $x \in W^{1,1}\left(\left[a, b_{*}\right], R^{n}\right)$.

According to Step 3, there exist $p$, real numbers $\lambda \geq 0$ and $\xi$ such that

(A4) $\lambda+\|p\|_{\infty}+|\xi|=1$

(B4) $\dot{p}(t) \in \operatorname{co}\left\{\alpha:(\alpha, \mathrm{p}(\mathrm{t})) \in \lambda \partial \mathrm{L}\left(\mathrm{t}, \mathrm{x}_{*}(\mathrm{t}), \dot{\mathrm{x}}_{*}(\mathrm{~s})\right)\right\}$ a.e. $t \in\left[a_{*}, b_{*}\right]$,

(C4) $\left(-\xi, p\left(a_{*}\right)\right) \in \lambda \partial g_{1}\left(a_{*}, x_{*}\left(a_{*}\right)\right),-p\left(b_{*}\right)=\lambda \nabla_{x} g_{2}\left(b_{*}, x_{*}\left(b_{*}\right)\right)$,

(D4) $\left\langle p(t), \dot{x}_{*}(t)\right\rangle-\lambda L\left(t, x_{*}(t), \dot{x}_{*}(t)\right) \geq\langle p(t), v\rangle-\lambda L\left(t, x_{*}(t), v\right)$ for all $v \in R^{n}$, a.e. $t \in\left[a_{*}, b_{*}\right]$, 
(E4)

$$
\xi \leq \lim _{\sigma \rightarrow 0} \operatorname{ess} \sup _{\left[a_{*}, a_{*}+\sigma\right]} \mathcal{H}_{\lambda}\left(t, x_{*}(t), \dot{x}_{*}(t), p\left(a_{*}\right)\right)
$$

Moreover,

$$
\xi \geq \lim _{\sigma \rightarrow 0} \operatorname{ess} \inf _{\left[a_{*}-\sigma, a_{*}\right]} \mathcal{H}_{\lambda}\left(t, x_{*}\left(a_{*}\right), \bar{u}(t), p\left(a_{*}\right)\right)
$$

Since $\left(\left[a_{*}, b_{*}\right], x_{*}\right)$ is also a solution of the problem

Minimize $g_{1}\left(a_{*}, x\left(a_{*}\right)\right)+g_{2}(b, x(b))+g_{3}(b)+\int_{a_{*}}^{b} L(t, x(t), \dot{x}(t)) d t$ over processes $\left(\left[a_{*}, b\right], x\right)$ which satisfy $x \in W^{1,1}\left(\left[a_{*}, b\right], R^{n}\right)$,

a similar argument as in Step 1 shows that

$$
\begin{aligned}
& -\lambda k_{3}+\lim _{\sigma \rightarrow 0} \operatorname{ess} \inf _{\left[b_{*}, b_{*}+\sigma\right]} \mathcal{H}_{\lambda}\left(t, x_{*}\left(b_{*}\right), \tilde{u}(t), p\left(b_{*}\right)\right) \leq \lambda \nabla_{b} g_{2}\left(b_{*}, x_{*}\left(b_{*}\right)\right) \leq \\
& \leq \lim _{\sigma \rightarrow 0} \operatorname{ess} \sup _{\left[b_{*}-\sigma, b_{*}\right]} \mathcal{H}_{\lambda}\left(t, x_{*}(t), \dot{x}_{*}(t), p\left(b_{*}\right)\right)+\lambda k_{3} .
\end{aligned}
$$

Step 5. We now return to the problem $(\mathrm{BP})$. Let $\left(\left[a_{*}, b_{*}\right], x_{*}\right)$ be a solution of (BP)

Minimize $J(a, b, x):=l(a, x(a), b, x(b))+\int_{a}^{b} L(t, x(t), \dot{x}(t)) d t$ over intervals $[a, b]$ and $\operatorname{arcs} x \in W^{1,1}\left([a, b], R^{n}\right)$.

We want to show that there exist $p$, real numbers $\lambda \geq 0, \xi$ and $\eta$ which satisfy the conclusion of Theorem 3.1.

Take a sequence $K_{i} \rightarrow \infty$. For each $i$ we put

$J_{i}(a, b, x, \tau, y)=l(a, x(a), \tau(a), y(a))+\int_{a}^{b} L(t, x(t), \dot{x}(t)) d t+K_{i}\left(|\tau(b)-b|^{2}+|y(b)-x(b)|^{2}\right)$,

where $\tau$ and $y$ are constant functions. Denote by $\tilde{W}$ the set of all $(a, b, z=(x, \tau, y))$ such that $x \in W^{1,1}\left([a, b], R^{n}\right), \tau \in R, y \in R^{n}$. It is clear that $\tilde{W}$ is a metric space with respect to metric $d$ induced by the norm

$$
|(a, b, x, \tau, y)|=|a|+|b|+|x(a)|+\left\|\dot{x}^{e}\right\|_{L^{1}}+|\tau|+|y| .
$$

Moreover, $J_{i}$ is continuous on $\tilde{W}$. Define a sequence $\epsilon_{i}$ by

$$
\epsilon_{i}^{2}:=J_{i}\left(a_{*}, b_{*}, x_{*}, b_{*}, x_{*}\left(b_{*}\right)\right)-\inf _{\tilde{W}} J_{i}(a, b, x, \tau, y) .
$$

We claim that $\epsilon_{i} \rightarrow 0$. In fact, from (BH1) we get

$$
l(a, x(a), \tau, y) \geq l(a, x(a), b, x(b))-k_{l}(|\tau-b|+|y-x(b)|) .
$$


Hence

$$
\begin{aligned}
J_{i}(a, b, x, \tau, y) & \geq l(a, x(a), b, x(b))+\int_{a}^{b} L(t, x(t), \dot{x}(t)) d t- \\
& -k_{l}(|\tau-b|+|y-x(b)|)+K_{i}\left(|\tau(b)-b|^{2}+|y(b)-x(b)|^{2}\right) \\
& \geq J_{i}\left(a_{*}, b_{*}, x_{*}, b_{*}, x_{*}\left(b_{*}\right)\right)-k_{l}^{2} / 2 K_{i} .
\end{aligned}
$$

This implies that $\epsilon_{i} \leq \frac{k_{l}}{\sqrt{2 K_{i}}} \rightarrow 0$. Since $\left(a_{*}, b_{*}, x_{*}, b_{*}, x_{*}\left(b_{*}\right)\right)$ is an $\epsilon_{i}$ minimizer, Ekeland's principle give us, for each $i$, a point $\left(a_{i}, b_{i}, x_{i}, \tau_{i}, y_{i}\right)$ such that

$$
\begin{gathered}
d\left[\left(a_{i}, b_{i}, x_{i}, \tau_{i}, y_{i}\right),\left(a_{*}, b_{*}, x_{*}, b_{*}, x_{*}\left(b_{*}\right)\right)\right] \leq \epsilon_{i}, \\
J_{i}\left(a_{i}, b_{i}, x_{i}, \tau_{i}, y_{i}\right) \leq J_{i}(a, b, x, \tau, y)+\epsilon_{i} d\left[(a, b, x, \tau, y),\left(a_{i}, b_{i}, x_{i}, \tau_{i}, y_{i}\right)\right] \forall(a, b, a, \tau, y) \in \tilde{W}
\end{gathered}
$$

From (4) we get $a_{i} \rightarrow a_{*}, b_{i} \rightarrow b_{*}, \tau_{i} \rightarrow b_{*}, y_{i} \rightarrow x_{*}\left(b_{*}\right), x_{i} \rightarrow x_{*}$ uniformly, $\dot{x}_{i}^{e} \rightarrow \dot{x}_{*}^{e}$ a.e. and in $L^{1}$. It follows from $(5)$ that $\left(a_{i}, b_{i}, x_{i}, \tau_{i}, y_{i}\right)$ is a $\tilde{W}$ minimizer of the functional

$$
\begin{aligned}
& \tilde{J}_{i}(a, z):=l(a, x(a), \tau(a), y(a))+\epsilon_{i}\left(\left|a-a_{i}\right|+\left|x(a)-x_{i}\left(a_{i}\right)\right|+\left|\tau-\tau_{i}\right|+\left|y-y_{i}\right|\right) \\
& +\epsilon_{i} \int_{a \wedge a_{i}}^{a}\left|\dot{x}_{i}^{e}(t)\right| d t+\int_{a}^{b}\left(L(t, x(t), \dot{x}(t))+\epsilon_{i}\left|\dot{x}(t)-\dot{x}_{i}^{e}(t)\right|\right) d t+ \\
& +\epsilon_{i} K_{i}\left(|\tau(b)-b|^{2}+|y(b)-x(b)|^{2}\right)+\epsilon_{i}\left(\left|b-b_{i}\right|+\int_{b}^{b \vee b_{i}}\left|\dot{x}_{i}^{e}(t)\right| d t\right),
\end{aligned}
$$

where $z:=(x, \tau, y)$. Note that since $\dot{x}_{i}^{e} \rightarrow \dot{x}_{*}^{e}$ in $L^{1}$, there exists $h \in L^{1}$ such that $\left|\dot{x}_{i}^{e}(t)\right| \leq h(t)$ a.e. Hence the functions $a \mapsto \int_{a \wedge a_{i}}^{a}\left|\dot{x}_{i}^{e}(t)\right| d t$ and $b \mapsto \int_{b}^{b \vee b_{i}}\left|\dot{x}_{i}^{e}(t)\right| d t$ are Lipschitz continuous with $\operatorname{rank} M=\operatorname{esssuph}$.

According to Step 4, there exist $p_{i}$, real numbers $\lambda_{i} \geq 0, \xi_{i}, \eta_{i}$ and $r_{i}$ such that

(A5) $\lambda_{i}+\left\|p_{i}\right\|_{\infty}+\left|\xi_{i}\right|+\left|\eta_{i}\right|+\left|r_{i}\right|=1$,

(B5) $\dot{p}_{i}(t) \in \operatorname{co}\left\{\alpha:\left(\alpha, \mathrm{p}_{\mathrm{i}}(\mathrm{t})\right) \in \lambda \partial \mathrm{L}\left(\mathrm{t}, \mathrm{x}_{\mathrm{i}}(\mathrm{t}), \dot{\mathrm{x}}_{\mathrm{i}}(\mathrm{t})\right)+\epsilon_{\mathrm{i}} \lambda_{\mathrm{i}}\{0\} \times \mathrm{B}\right\}$ a.e. $t \in\left[a_{i}, b_{i}\right]$,

(C5) $\left(-\xi_{i}, p_{i}\left(a_{i}\right), \eta_{i}, r_{i}\right) \in \lambda_{i} \partial l\left(a_{i}, x_{i}\left(a_{i}\right), \tau_{i}, y_{i}\right)+\lambda_{i} \epsilon_{i} M(B \times\{0\} \times\{0\} \times\{0\})+\lambda_{i} \epsilon_{i} B^{4}$ and $-\left(p_{i}\left(b_{i}\right), \eta_{i}, r_{i}\right)=2 \epsilon_{i} K_{i}\left(-y_{i}+x_{i}\left(b_{i}\right), \tau_{i}-b_{i}, y_{i}-x_{i}\left(b_{i}\right)\right)$

(D5) $\left\langle p_{i}(t), \dot{x}_{i}(t)\right\rangle-\lambda_{i} L\left(t, x_{i}(t), \dot{x}_{i}(t)\right) \geq\left\langle p_{i}(t), v\right\rangle-\lambda L\left(t, x_{i}(t), v\right)-\lambda_{i} \epsilon_{i}\left|v-\dot{x}_{i}\right|$ for all $v \in R^{n}$ and a.e. $t \in\left[a_{i}, b_{i}\right]$.

(E5)

$$
\begin{aligned}
& \lim _{\sigma \rightarrow 0} \operatorname{ess} \inf _{\left[a_{i}-\sigma, a_{i}\right]}\left[\left\langle p_{i}\left(a_{i}\right), \bar{u}_{i}(t)\right\rangle-\lambda_{i} L\left(t, x_{i}(t), \dot{x}_{i}(t)\right)-\lambda_{i} \epsilon_{i}\left|\bar{u}_{i}(t)\right|\right] \leq \xi_{i} \leq \\
& \leq \lim _{\sigma \rightarrow 0} \operatorname{ess} \sup _{\left[a_{i}, a_{i}+\sigma\right]}\left[\left\langle p_{i}\left(a_{i}\right), \dot{x}_{i}(t)\right\rangle-\lambda_{i} L\left(t, x_{i}(t), \dot{x}_{i}(t)\right)\right]
\end{aligned}
$$

and 


$$
\begin{aligned}
& -\lambda_{i} \epsilon_{i}(1+M)+\lim _{\sigma \rightarrow 0} \operatorname{ess} \inf _{\left[b_{i}, b_{i}+\sigma\right]}\left[\left\langle p_{i}\left(b_{i}\right), \tilde{u}_{i}(t)\right\rangle-\lambda_{i} L\left(t, x_{i}(t), \tilde{u}_{i}(t)\right)-\lambda_{i} \epsilon_{i}\left|\tilde{u}_{i}(t)\right|\right] \leq \\
& \leq 2 K_{i} \epsilon_{i}\left(b_{i}-\tau_{i}\right)=\eta_{i} \leq \lim _{\sigma \rightarrow 0} \operatorname{ess} \sup _{\left[b_{i}-\sigma, b_{i}\right]}\left[\left\langle p_{i}\left(b_{i}\right), \dot{x}_{i}(t)\right\rangle-\lambda L\left(t, x_{i}(t), \dot{x}_{i}(t)\right)\right]+\lambda_{i} \epsilon_{i}(1+M),
\end{aligned}
$$

where $\bar{u}_{i}=\bar{u}^{e}$ and $\tilde{u}_{i}=\tilde{u}^{e}$.

Since $p_{i}$ 's are bounded and their derivatives are bounded by an integrable function, $p_{i} \rightarrow p$ uniformly and $\dot{p}_{i} \rightarrow \dot{p}$ weakly in $L^{1}$ for some $p \in W^{1,1}$. A further subsequence extraction ensures that $\lambda_{i} \rightarrow \lambda, \eta_{i} \rightarrow \eta, \xi_{i} \rightarrow \xi$ and $r_{i} \rightarrow-p\left(b_{*}\right)$. Note that since $-p_{i}\left(b_{i}\right)=r_{i}$, it follows that that $\lambda+\|p\| \neq 0$. By passing to the limit and standard arguments we can show that $\lambda$ and $p$ satisfy the conclusion of the theorem. The proof of the theorem is complete.

Remark 3.2 Theorem 8.4.1 in [22] gave necessary conditions for problem $(\mathrm{P})$ in the scalar case. It is possible to reduce $(\mathrm{BP})$ to $(\mathrm{P})($ in the case $m=1)$. However, it seems that this transformation causes the structure of the problem becoming poor and so it is difficult to obtain the desired conclusions. In the above argument, we exploited the structure of (BP) and gave a direct proof.

\section{Necessary conditions for MOC}

In this section we derive necessary conditions for $(\mathrm{P})$. Fix a feasible triple $\left(\left[a_{*}, b_{*}\right], x_{*}\right)$ and assume the following hypotheses which involve positive number $\delta$, a nonnegative function $k_{F} \in L^{1}\left[a_{*}, b_{*}\right]$ and a number $\beta \geq 0$ :

(H1) $g$ is Lipschitz continuous on a neighborhood of $\left(a_{*}, x_{*}\left(a_{*}\right), b_{*}, x_{*}\left(b_{*}\right)\right)$ with rank $k_{g}$ and $C$ is a closed set.

(H2) $F$ is $\mathcal{L} \times \mathcal{B}$ measurable with nonempty values and $\operatorname{Gph} F(t, \cdot)$ is closed.

(H3) $F$ has the integrable sub-Lipschizian property (see [10]), that is,

$$
F\left(t, x^{\prime}\right) \cap\left(x_{*}(t)+N B\right) \subset F(t, x)+\left(k_{F}(t)+\beta N\right)\left|x^{\prime}-x\right| B
$$

for all $N \geq 0, x^{\prime}, x \in x_{*}(t)+\delta B$, a.e. $t \in\left[a_{*}, b_{*}\right]$.

(H4) There exist positive constants $c_{0}, c_{1}, k_{0}$ and $k_{1}$ such that

$$
\left\{\begin{array}{l}
F(t, x) \subset c_{0} B \\
F\left(t, x^{\prime}\right) \subset F(t, x)+k_{0}\left|x^{\prime}-x\right| B,
\end{array}\right.
$$

for a.e. $t \in\left[a_{*}-\delta, a_{*}\right]$ and for all $x, x^{\prime} \in x_{*}\left(a_{*}\right)+\delta B$;

$$
\left\{\begin{array}{l}
F(t, x) \subset c_{1} B \\
F\left(t, x^{\prime}\right) \subset F(t, x)+k_{1}\left|x^{\prime}-x\right| B
\end{array}\right.
$$


for a.e. $t \in\left[b_{*}, b_{*}+\delta\right]$ and for all $x, x^{\prime} \in x_{*}\left(b_{*}\right)+\delta B$.

In what follows $H(t, x, p):=\sup \{\langle p, v\rangle: v \in F(t, x(t))\}$ and $\operatorname{ess}_{\tau \rightarrow t} f(\tau)$ is the essential value of a real value function $f$ at $t \in I \subset R$, that is, $\operatorname{ess}_{\tau \rightarrow t} f(\tau):=\left[a_{-}, a_{+}\right]$, where

$$
a_{-}:=\lim _{\delta \rightarrow 0} \operatorname{ess} \inf _{\tau \in[t-\delta, t+\delta]} f(\tau) \text { and } a_{+}:=\lim _{\delta \rightarrow 0} \operatorname{ess} \sup _{\tau \in[t-\delta, t+\delta]} f(\tau) .
$$

We refer the reader to [22, Proposition 8.3.2] for properties of essential values.

We are ready to state our main result

Theorem 4.1 Suppose $x_{*}$ is a $W^{1,1}$ local minimizer of $(P)$, preference $\prec$ is regular at $g\left(a_{*}, x_{*}\left(a_{*}\right), b_{*}, x_{*}\left(b_{*}\right)\right)$ and assumptions (H1)- (H4) are satisfied. Then there exist an arc $p \in W^{1,1}\left(\left[a_{*}, b_{*}\right], R^{n}\right)$, a vector $w \in N_{\overline{\mathcal{L}}\left[g\left(a_{*}, x_{*}(a), b_{*}, x_{*}(b)\right)\right]}\left(g\left(a_{*}, x_{*}\left(a_{*}\right), b_{*}, x_{*}\left(b_{*}\right)\right)\right.$ with $|w|=1$ and real numbers $\lambda \geq 0, \xi$ and $\eta$ such that

(i) $\lambda+\|p\|_{\infty}=1$,

(ii) $\dot{p}(t) \in \operatorname{co}\left\{\alpha:(\alpha, p(t)) \in N_{\mathrm{Gph} F(t, \cdot)}\left(x_{*}(t), \dot{x}_{*}(t)\right)\right\}$ a.e. $t \in\left[a_{*}, b_{*}\right]$,

(iii) $\left(-\xi, p\left(a_{*}\right), \eta,-p\left(b_{*}\right)\right) \in \lambda \partial\left\langle w, g\left(a_{*}, x_{*}\left(a_{*}\right), b_{*}, x_{*}(b)\right)\right\rangle+N_{C}\left(a_{*}, x_{*}\left(a_{*}\right), b_{*}, x_{*}\left(b_{*}\right)\right)$,

(iv) $\left\langle p(t), \dot{x}_{*}(t)\right\rangle=H\left(t, x_{*}(t), p(t)\right)$, a.e. $t \in\left[a_{*}, b_{*}\right]$,

(v) $\xi \in \operatorname{ess}_{t \rightarrow a_{*}} H\left(t, x_{*}\left(a_{*}\right), p\left(a_{*}\right)\right)$ and $\eta \in \operatorname{ess}_{t \rightarrow b_{*}} H\left(t, x_{*}\left(b_{*}\right), p\left(b_{*}\right)\right)$.

Proof. Define a mapping $\rho_{F}: R \times R^{n} \times R^{n} \rightarrow R$ by

$$
\rho_{F}(t, x, \dot{x})=\inf \{|\dot{x}-v|: v \in F(t, x)\} .
$$

According to Lemma 7 in [24] it follows from (H3) that $\rho_{F}(t, \cdot, \cdot)$ satisfies condition (BH3) for a.e. $t \in\left[a_{*}, b_{*}\right]$. Put

$$
W_{\epsilon}=\left\{([a, b], x): x \in W^{1,1}([a, b]), d\left(([a, b], x),\left[a_{*}, b_{*}\right], x_{*}\right) \leq \epsilon\right\}
$$

and

$$
S_{\epsilon}=\left\{([a, b], x) \in W_{\epsilon}:(a, x(a), b, x(b)) \in C, \dot{x}(t) \in F(t, x(t)), \text { a.e. }\right\} .
$$

It is clear that $W_{\epsilon}$ is a complete metric space and $S_{\epsilon}$ is a closed set in $W_{\epsilon}$.

Fix $N$ and reduce the size of $\epsilon$ such that $\left(\left[a_{*}, b_{*}\right], x_{*}\right)$ is the solution of $(\mathrm{P})$ in $S_{\epsilon}$.

As in [24] and [2], we use a variant of Ioffe's scheme [8] by considering two following possible situation:

(a) There exist $\epsilon^{\prime} \in(0, \epsilon)$ and $K>0$ such that for any $([a, b], x) \in W_{\epsilon^{\prime}}$, one has

$$
d\left(([a, b], x), S_{\epsilon}\right) \leq K\left[\int_{a}^{b} \rho_{F}(t, x(t), \dot{x}(t)) d t+K d_{C}(a, x(a), b, x(b))\right] .
$$

(b) There exist a sequence $\epsilon_{k}^{\prime} \rightarrow 0$, a sequence $\left(\left[a_{k}, b_{k}\right], x_{k}\right) \in W_{\epsilon_{k}^{\prime}}$ such that

$$
d\left(\left(\left[a_{k}, b_{k}\right], x_{k}\right), S_{\epsilon}\right)>2 k\left[\int_{a_{k}}^{b_{k}} \rho_{F}\left(t, x_{k}(t), \dot{x}_{k}(t)\right) d t+2 k d_{C}\left(a_{k}, x_{k}\left(a_{k}\right), b_{k}, x_{k}\left(b_{k}\right)\right)\right] .
$$


Case (a) Since $g\left(a_{*}, x_{*}(a), b_{*}, x_{*}\left(b_{*}\right)\right) \in \overline{\mathcal{L}}\left[g\left(a_{*}, x_{*}\left(a_{*}\right), b_{*}, x_{*}\left(b_{*}\right)\right)\right]$, there exists a sequence $\theta_{k} \in \mathcal{L}\left[g\left(a_{*}, x_{*}\left(a_{*}\right), b_{*}, x_{*}\left(b_{*}\right)\right)\right]$ such that $\left|\theta_{k}-g\left(a_{*}, x_{*}\left(a_{*}\right), b_{*}, x_{*}\left(b_{*}\right)\right)\right| \leq 1 / k^{2}$. Put $\Omega_{k}=$ $\overline{\mathcal{L}}\left[\theta_{k}\right]$ and define the function

$$
\varphi(a, b, x, \theta)= \begin{cases}|g(a, x(a), b, x(b))-\theta| & \text { if }(a, b, x, \theta) \in S_{\epsilon^{\prime}} \times \Omega_{k} \\ +\infty & \text { otherwise. }\end{cases}
$$

We claim that $\varphi$ is l.s.c. on $W_{\epsilon^{\prime}} \times \Omega_{k}$. Indeed, assume that $((a, b, x), \theta) \in W_{\epsilon^{\prime}} \times \Omega_{k}$ and $\left(\left(a_{n}, b_{n}, x_{n}\right), \theta_{n}\right) \stackrel{W_{\epsilon^{\prime}}}{\longrightarrow}((a, b, x), \theta)$. If $(a, b, x, \theta) \in S_{\epsilon^{\prime}} \times \Omega_{k}$, then it follows from Lipschitzian continuity of $g$ that

$$
\begin{aligned}
\left|\varphi\left(a_{n}, b_{n}, x_{n}, \theta_{n}\right)-\varphi(a, b, x, \theta)\right| & \leq k_{g}\left(\left|a_{n}-a\right|+\left|b_{n}-b\right|+\left|x_{n}\left(a_{n}\right)-x(a)\right|\right. \\
& \left.+\left|x_{n}\left(b_{n}\right)-x(b)\right|\right)+\left|\theta_{n}-\theta\right| \\
& \leq k_{g}\left(\left|a_{n}-a\right|+\left|b_{n}-b\right|+2\left\|x_{n}-x\right\|_{\infty}\right)+\left|\theta_{n}-\theta\right| \rightarrow 0
\end{aligned}
$$

If $(a, b, x, \theta) \notin S_{\epsilon^{\prime}} \times \Omega_{k}$ then $\left(a_{n}, b_{n}, x_{n}, \theta_{n}\right) \notin S_{\epsilon^{\prime}} \times \Omega_{k}$ for $n$ sufficiently large because $S_{\epsilon^{\prime}} \times \Omega_{k}$ is closed in $W_{\epsilon^{\prime}} \times R^{m}$. Hence $\lim _{n \rightarrow \infty} \varphi\left(a_{n}, b_{n}, x_{n}, \theta_{n}\right)=+\infty \geq \varphi(a, b, x, \theta)$. Thus $\varphi$ is l.s.c. Since $\varphi(a, b, x, \theta) \geq 0$, one has

$$
\varphi\left(a_{*}, b_{*}, x_{*}, \theta_{k}\right) \leq \inf _{(x, \theta) \in W_{\epsilon^{\prime}} \times \Omega_{k}} \varphi(a, b, x, \theta)+1 / k^{2} .
$$

The Ekeland principle gives us, for each $k$, a point $\left(a_{k}, b_{k}, x_{k}, \chi_{k}\right) \in W_{\epsilon^{\prime}} \times \Omega_{k}$ such that

$$
\begin{gathered}
\varphi\left(a_{k}, b_{k}, x_{k}, \chi_{k}\right) \leq \varphi\left(a_{*}, b_{*}, x_{*}, \theta_{k}\right)<\frac{1}{k^{2}} \\
\left|a_{k}-a_{*}\right|+\left|b_{k}-b_{*}\right|+\left|x_{k}\left(a_{k}\right)-x_{*}\left(a_{*}\right)\right|+\left\|\dot{x}_{k}^{e}-\dot{x}_{*}^{e}\right\|_{L^{1}}+\left|\chi_{k}-\theta_{k}\right| \leq 1 / k, \\
\left.\varphi\left(a_{k}, b_{k}, x_{k}, \chi_{k}\right) \leq \varphi(a, b, x, \theta)+\frac{1}{k} d\left(([a, b], x),\left(\left[a_{k}, b_{k}\right], x_{k}\right)\right)+\left|\theta-\chi_{k}\right|\right)
\end{gathered}
$$

for all $((a, b, x), \theta) \in W_{\epsilon^{\prime}} \times \Omega_{k}$. From (7), we get $\left(a_{k}, b_{k}, x_{k}\right) \in S_{\epsilon^{\prime}}$. (8) implies that $a_{k} \rightarrow a_{*}, b_{k} \rightarrow b_{*}, x_{k}\left(a_{k}\right) \rightarrow x_{*}\left(a_{*}\right), x_{k}^{e} \rightarrow x_{*}^{e}$ uniformly, $\dot{x}_{k}^{e} \rightarrow \dot{x}_{*}^{e}$ a.e. and $\chi_{k} \rightarrow$ $g\left(a_{*}, x_{*}\left(a_{*}\right), b_{*}, x_{*}\left(b_{*}\right)\right)$. We claim that $\chi_{k} \neq g\left(a_{k}, x_{k}\left(a_{k}\right), b_{k}, x_{k}\left(b_{k}\right)\right)$. Indeed, suppose that $\chi_{k}=g\left(a_{k}, x_{k}\left(a_{k}\right), b_{k}, x_{k}\left(b_{k}\right)\right)$. Since $\prec$ is closed, the relation $\chi_{k} \in \overline{\mathcal{L}}\left[\chi_{k}\right]$ and $\chi_{k} \prec$ $g\left(a_{*}, x_{*}\left(a_{*}\right), b_{*}, x_{*}\left(b_{*}\right)\right)$ imply that

$$
g\left(a_{k}, x_{k}\left(a_{k}\right), b_{k}, x_{k}\left(b_{k}\right)\right)=\chi_{k} \prec g\left(a_{*}, x_{*}\left(a_{*}\right), b_{*}, x_{*}\left(b_{*}\right)\right) .
$$

This contradicts to the fact that $\left(\left[a_{*}, b_{*}\right], x_{*}\right)$ is a minimizer.

Put $w_{k}=\frac{\chi_{k}-g\left(a_{k}, x_{k}\left(a_{k}\right), b_{k}, x_{k}\left(b_{k}\right)\right)}{\left|\chi_{k}-g\left(a_{k}, x_{k}\left(a_{k}\right), b_{k}, x_{k}(b)\right)\right|}$. We can assume that $w_{k} \rightarrow w$ with $|w|=1$. Substituting $(a, b, x)=\left(a_{k}, b_{k}, x_{k}\right)$ into $(9)$, it follows that

$$
\left.0 \in \partial\left(\left|g\left(a_{k}, x_{k}\left(a_{k}\right), b_{k}, x_{k}\left(b_{k}\right)\right)-\cdot\right|\right)+\frac{1}{k}\left|\cdot-\chi_{k}\right|\right)\left(\chi_{k}\right)+N_{\Omega_{k}}\left(\chi_{k}\right) .
$$


This implies that $w_{k} \in \frac{1}{k} B+N_{\Omega_{k}}\left(\chi_{k}\right)$. Hence

$$
w \in \lim _{k \rightarrow \infty} N_{\Omega_{k}}\left(\chi_{k}\right) \subset N_{\overline{\mathcal{L}}\left[g\left(a_{*}, x_{*}\left(a_{*}\right), b_{*}, x_{*}\left(b_{*}\right)\right)\right]}\left(g\left(a_{*}, x_{*}\left(a_{*}\right), b_{*}, x_{*}\left(b_{*}\right)\right) .\right.
$$

Also, substituting $\theta=\chi_{k}$ into (9), it follows that

$$
\varphi\left(a_{k}, b_{k}, x_{k}, \chi_{k}\right) \leq \varphi\left(a, b, x, \chi_{k}\right)+\frac{1}{k} d\left(([a, b], x),\left(\left[a_{k}, b_{k}\right], x_{k}\right)\right) .
$$

Combining this with (5) yields

$$
\begin{aligned}
\varphi\left(a_{k}, b_{k}, x_{k}, \chi_{k}\right) & \leq \varphi\left(a, b, x, \chi_{k}\right)+\frac{1}{k} d\left(([a, b], x),\left(\left[a_{k}, b_{k}\right], x_{k}\right)\right)+ \\
& +\left[\int_{a}^{b} \rho_{F}(t, x(t), \dot{x}(t)) d t+K d_{C}(a, x(a), b, x(b))\right] .
\end{aligned}
$$

for all $([a, b], x) \in W_{\epsilon^{\prime}}$. This implies that $\left(\left[a_{k}, b_{k}\right], x_{k}\right)$ is a $W_{\epsilon^{\prime}}$ minimizer of the Bolza problem:

$$
\begin{aligned}
J(a, b, x):= & \int_{a}^{b}\left(\rho_{F}(t, x(t), \dot{x}(t))+\frac{1}{k}\left|\dot{x}-\dot{x}_{k}^{e}\right|\right) d t+\left|g(a, x(a), b, x(b))-\chi_{k}\right|+ \\
& +K d_{C}(a, x(a), b, x(b))+\frac{1}{k}\left(\left|a-a_{k}\right|+\left|b-b_{k}\right|+\left|x(a)-x_{k}\left(a_{k}\right)\right|+\right. \\
& +\int_{a_{k} \wedge a}^{a}\left|\dot{x}_{k}^{e}\right| d t+\int_{b}^{b \vee b_{k}}\left|\dot{x}_{k}^{e}\right| d t
\end{aligned}
$$

Put

$$
\begin{gathered}
L(t, x(t), \dot{x}(t))=\rho_{F}(t, x(t), \dot{x}(t))+\frac{1}{k}\left|\dot{x}-\dot{x}_{k}^{e}\right|, \\
l(a, x(a), b, x(b))=\left|g(a, x(a), b, x(b))-\chi_{k}\right|+K d_{C}(a, x(a), b, x(b))+ \\
+\frac{1}{k}\left(\left|a-a_{k}\right|+\left|b-b_{k}\right|+\left|x(a)-x_{k}\left(a_{k}\right)\right|+\int_{a_{k} \wedge a}^{a}\left|\dot{x}_{k}^{e}\right| d t+\int_{b}^{b \vee b_{k}}\left|\dot{x}_{k}^{e}\right| d t\right) .
\end{gathered}
$$

It is easy to check that hypotheses (BH1)-(BH3) hold for $l$ and $L$. By Theorem 3.1, there exist $p_{k} \in W^{1,1}$, real numbers $\lambda_{k} \geq 0, \xi_{k}, \eta_{k}$ such that

(A) $\lambda_{k}+\left|p_{k}\right|_{\infty}=1$

(B) $\dot{p}_{k}(t) \in \operatorname{co}\left\{\alpha:\left(\alpha, p_{k}(t)\right) \in \lambda_{k} \partial \rho_{F}\left(t, x_{k}(t), \dot{x}_{k}(t)\right)+\frac{\lambda_{k}}{k}\{0\} \times B\right\}$ a.e. $t \in\left[a_{k}, b_{k}\right]$.

(C) $\left(-\xi_{k}, p_{k}\left(a_{k}\right), \eta_{k},-p_{k}\left(b_{k}\right)\right) \in \lambda_{k} \partial\left\langle w_{k}, g\left(a_{k}, x_{k}\left(a_{k}\right), b_{k}, x_{k}\left(b_{k}\right)\right)\right\rangle+\frac{\lambda_{k}}{k} B^{3} \times\{0\}+\frac{\lambda_{k}}{k} M(B \times$

$\{0\} \times B \times\{0\})+\lambda_{k} K \partial d_{C}\left(a_{k}, x_{k}\left(a_{k}\right), b_{k}, x_{k}\left(b_{k}\right)\right)$.

(D) $\left\langle p_{k}(t), \dot{x}_{k}(t)\right\rangle-\lambda_{k} \rho_{F}\left(t, x_{k}(t), \dot{x}_{k}(t)\right) \geq\left\langle p_{k}(t), \dot{v}\right\rangle-\lambda_{k} \rho_{F}\left(t, x_{k}(t), v\right)-\frac{\lambda_{k}}{k}\left|v-\dot{x}_{k}^{e}\right|$ for all $v \in R^{n}$ a.e.,

(E)

$$
\xi_{k} \leq \lim _{\sigma \rightarrow 0} \operatorname{ess} \sup _{t \in\left[a_{k}-\sigma, a_{k}\right]}\left(\left\langle p_{k}\left(a_{k}\right), \dot{x}_{k}(t)\right\rangle-\lambda_{k} \rho_{F}\left(t, x_{k}(t), \dot{x}_{k}(t)\right)\right.
$$


and

$$
\eta_{k} \leq \lim _{\sigma \rightarrow 0} \operatorname{ess} \sup _{t \in\left[b_{k}, b_{k}+\sigma\right]}\left(\left\langle p_{k}\left(b_{k}\right), \dot{x}_{k}(t)\right\rangle-\lambda_{k} \rho_{F}\left(t, x_{k}(t), \dot{x}_{k}(t)\right) .\right.
$$

Fix any $\sigma<\delta$. By (H4) we can find essentially bounded selections $\bar{u}_{k}$ and $\tilde{u}_{k}$ of $F\left(\cdot, x_{k}\left(a_{k}\right)\right)$ and $F\left(\cdot, x_{k}\left(b_{k}\right)\right)$, respectively such that

$$
\left\langle p_{k}\left(a_{k}\right), \bar{u}_{k}(t)\right\rangle=\max _{u \in F\left(t, x_{k}\left(a_{k}\right)\right)}\left\langle p_{k}\left(a_{k}\right), u\right\rangle=H\left(t, x_{k}\left(a_{k}\right), p_{k}\left(a_{k}\right)\right) \text { a.e. } t \in\left[a_{k}-\sigma, a_{k}\right]
$$

and

$$
\left\langle p_{k}\left(b_{k}\right), \tilde{u}_{k}(t)\right\rangle=\max _{u \in F\left(t, x_{k}\left(b_{k}\right)\right)}\left\langle p_{k}\left(b_{k}\right), u\right\rangle=H\left(t, x_{k}\left(b_{k}\right), p_{k}\left(b_{k}\right)\right) \text { a.e. } t \in\left[b_{k}, b_{k}+\sigma\right] .
$$

Since $L\left(t, x_{k}\left(a_{k}\right), \bar{u}_{k}(t)\right)=\frac{1}{k}\left|\bar{u}_{k}(t)\right|$, the function $t \mapsto L\left(t, x_{k}\left(a_{k}\right), \bar{u}_{k}(t)\right)$ is essentially bounded on $\left[a_{k}-\sigma, a_{k}\right]$. Moreover the function $x \mapsto L(t, x, u)$ is Lipschitz continuous with rank $k_{0}$ in a neighborhood $x_{k}\left(a_{k}\right)$ for $k$ sufficiently large and for a.e. $t \in\left[a_{k}-\sigma, a_{k}\right]$. Also, the function $t \mapsto L\left(t, x_{k}\left(b_{k}\right), \tilde{u}_{k}(t)\right)$ is essentially bounded on $\left[b_{k}, b_{k}+\sigma\right]$ and $x \mapsto L(t, x, u)$ is Lipschitz continuous with rank $k_{1}$ for a.e. $t \in\left[b_{k}, b_{k}+\sigma\right]$. Hence (BH4) is fulfilled. By the conclusion of Theorem 3.1 we have

$$
\xi_{k} \geq \lim _{\sigma \rightarrow 0} \operatorname{ess} \inf _{t \in\left[a_{k}-\sigma, a_{k}\right]}\left(H\left(t, x_{k}\left(a_{k}\right), p_{k}\left(a_{k}\right)\right)-\frac{\lambda_{k}}{k}\left|\bar{u}_{k}(t)\right|\right)
$$

and

$$
\eta_{k} \geq \lim _{\sigma \rightarrow 0} \operatorname{ess} \inf _{t \in\left[b_{k}, b_{k}+\sigma\right]}\left(H\left(t, x_{k}\left(b_{k}\right), p_{k}\left(b_{k}\right)\right)-\frac{\lambda_{k}}{k}\left|\tilde{u}_{k}(t)\right|\right) .
$$

Since $p_{k}$ 's are bounded and their derivatives are bounded by an integrable function, $p_{k} \rightarrow p$ uniformly and $\dot{p}_{k} \rightarrow \dot{p}$ weakly in $L^{1}$ for some $p \in W^{1,1}$. A further subsequence extraction ensures that $\lambda_{k} \rightarrow \lambda, \eta_{k} \rightarrow \eta, \xi_{k} \rightarrow \xi$.

By passing to the limit as $k \rightarrow \infty$ in (A) we obtain (i). From (B) and Lemma 2.1, we have

$$
\dot{p}_{k}(t) \in \operatorname{co}\left\{\alpha:\left(\alpha, p_{k}(t)\right) \in N_{\operatorname{Grph} F(t, \cdot)}\left(x_{k}(t), \dot{x}_{k}(t)\right)+\frac{\lambda_{k}}{k}\{0\} \times B\right\} .
$$

Passing to the limit as $k \rightarrow \infty$ yields

$$
\dot{p}(t) \in \operatorname{co}\left\{\alpha:(\alpha, p(t)) \in N_{\operatorname{Grph} F(t, \cdot)}\left(x_{*}(t), \dot{x}_{*}(t)\right)\right\} .
$$

Hence $(i i)$ follows. As

$$
\lambda_{k} K \partial d_{C}\left(a_{k}, x_{k}\left(a_{k}\right), b_{k}, x_{k}\left(b_{k}\right)\right) \subset N_{C}\left(\left(a_{k}, x_{k}\left(a_{k}\right), b_{k}, x_{k}\left(b_{k}\right)\right),\right.
$$

passing to the limit in (C) and (D), we obtain (iii) and (iv), respectively.

By Lemma 2.2, $H(t, \cdot, \cdot)$ is continuous for a.e. $t$. Passing to the limit in (10) and (14), and using properties of essential values (see [22, Proposition 8.3.2]), we get

$$
\lim _{\sigma \rightarrow 0} \operatorname{ess} \inf _{t \in\left[a_{*}-\sigma, a_{*}\right]} H\left(t, x_{*}\left(a_{*}\right), p\left(a_{*}\right)\right) \leq \xi \leq \lim _{\sigma \rightarrow 0} \operatorname{ess} \sup _{t \in\left[a_{*}-\sigma, a_{*}\right]}\left\langle p\left(a_{*}\right), \dot{x}_{*}(t)\right\rangle .
$$


By (H4), we have

$$
F\left(t, x_{*}(t)\right) \subset F\left(t, x_{*}\left(a_{*}\right)\right)+k_{0} \| x_{*}(t)-x_{*}\left(a_{*}\right) \mid B \text { for a.e.t } \in\left[a_{*}-\sigma, a_{*}\right] .
$$

Hence

$$
\sup _{u \in F\left(t, x_{*}(t)\right)}\left\langle p\left(a_{*}\right), u\right\rangle \leq \sup _{u \in F\left(t, x_{*}\left(a_{*}\right)\right)}\left(\left\langle p\left(a_{*}\right), u\right\rangle+k_{0}\left|x_{*}(t)-x_{*}\left(a_{*}\right)\right|\right) .
$$

This implies that

$$
\lim _{\sigma \rightarrow 0} \operatorname{ess} \sup _{t \in\left[a_{*}-\sigma, a_{*}\right]} H\left(t, x_{*}(t), p\left(a_{*}\right)\right) \leq \lim _{\sigma \rightarrow 0} \operatorname{ess} \sup _{t \in\left[a_{*}-\sigma, a_{*}\right]} H\left(t, x_{*}\left(a_{*}\right), p\left(a_{*}\right)\right) .
$$

Combining (16) with (17) yields

$$
\lim _{\sigma \rightarrow 0} \operatorname{ess} \inf _{t \in\left[a_{*}-\sigma, a_{*}+\sigma\right]} H\left(t, x_{*}\left(a_{*}\right), p\left(a_{*}\right)\right) \leq \xi \leq \lim _{\sigma \rightarrow 0} \operatorname{ess} \sup _{t \in\left[a_{*}-\sigma, a_{*}+\sigma\right]} H\left(t, x_{*}\left(a_{*}\right), p\left(a_{*}\right)\right)
$$

which means that $\xi \in \operatorname{ess}_{t \rightarrow a_{*}} H\left(t, x_{*}\left(a_{*}\right), p\left(a_{*}\right)\right)$. By similar arguments, we can show that $\eta \in \operatorname{ess}_{t \rightarrow b_{*}} H\left(t, x_{*}\left(b_{*}\right), p\left(b_{*}\right)\right)$. Thus $(v)$ follows.

Case (b) Putting $\epsilon_{k}=d\left[\left(a_{k}, b_{k}, x_{k}\right), S_{\epsilon}\right]$, we have

$$
0<\epsilon_{k} \leq d\left(\left(\left[a_{k}, b_{k}\right], x_{k}\right),\left(\left[a_{*}, b_{*}\right], x_{*}\right)\right) \leq \epsilon_{k}^{\prime} \rightarrow 0 .
$$

Form (6) it follows that

$$
\inf _{(a, b, x) \in W_{\epsilon}} \tilde{J}(a, b, x)+\frac{\epsilon_{k}}{2 k}>\tilde{J}\left(a_{k}, b_{k}, x_{k}\right)
$$

where $\tilde{J}(a, b, x):=\int_{a}^{b} \rho_{F}(t, x(t), \dot{x}(t)) d t+2 k d_{C}(a, x(a), b, x(b))$. By the Ekeland principle, for each $k$ there exists a triple $\left(\left[\bar{a}_{k}, \bar{b}_{k}\right], \bar{x}_{k}\right) \in W_{\epsilon}$ such that

$$
d\left(\left(\left[\bar{a}_{k}, \bar{b}_{k}\right], \bar{x}_{k}\right),\left(\left[a_{k}, b_{k}\right], x_{k}\right)\right) \leq \epsilon_{k} / 2
$$

and $\left(\left[\bar{a}_{k}, \bar{b}_{k}\right], \bar{x}_{k}\right)$ is a $W_{\epsilon}$ minimizer of the functional

$$
J_{*}(a, b, x):=\tilde{J}(a, b, x)+\frac{1}{k} d\left(([a, b], x),\left(\left[\bar{a}_{k}, \bar{b}_{k}\right], \bar{x}_{k}\right)\right) .
$$

It is clear that $(18)$ implies $\left(\left[\bar{a}_{k}, \bar{b}_{k}\right], \bar{x}_{k}\right) \stackrel{W_{\epsilon}}{\longrightarrow}\left(\left[a_{*}, b_{*}\right], x_{*}\right)$ and $\left(\left[\bar{a}_{k}, \bar{b}_{k}\right], \bar{x}_{k}\right) \notin S_{\epsilon}$. Rewrite (19) in the form

$$
\begin{aligned}
J_{*}(a, b, x)= & \int_{a}^{b} \rho_{F}(t, x(t), \dot{x}(t))+\left|\dot{x}(t)-\dot{\bar{x}}^{e}(t)\right| d t+2 k d_{C}(a, x(a), b, x(b))+ \\
& +\frac{1}{k}\left(\left|a-\bar{a}_{k}\right|+\left|b-\bar{b}_{k}\right|+\int_{a \wedge a_{k}}^{a}\left|\dot{\bar{x}}_{k}^{e}\right| d t+\int_{b}^{b \vee b_{k}}\left|\dot{\bar{x}}_{k}^{e}\right| d t\right) .
\end{aligned}
$$


According to Theorem 3.1, there exist $p_{k}$, real numbers $\lambda_{k} \geq 0, \xi_{k}$ and $\eta_{k}$ such that

$\left(\mathrm{A}^{\prime}\right) \lambda_{k}+\left|p_{k}\right|_{\infty}=1$,

$\left(\mathrm{B}^{\prime}\right) \dot{p}_{k}(t) \in \operatorname{co}\left\{\alpha:\left(\alpha, p_{k}(t)\right) \in \lambda_{k} \partial \rho_{F}\left(t, \bar{x}_{k}(t), \dot{\bar{x}}_{k}(t)\right)+\frac{\lambda_{k}}{k}\{0\} \times B\right\}$ a.e. $t \in\left[\bar{a}_{k}, \bar{b}_{k}\right]$.

(C') $\left(-\xi_{k}, p_{k}\left(\bar{a}_{k}\right), \eta_{k},-p_{k}\left(\bar{b}_{k}\right)\right) \in \lambda_{k} 2 k \partial d_{C}\left(\bar{a}_{k}, \bar{x}_{k}\left(a_{k}\right), \bar{b}_{k}, \bar{x}_{k}\left(\bar{b}_{k}\right)\right)+\frac{\lambda_{k}}{k} B^{2} \times\{0\} \times\{0\}+$ $\frac{\lambda_{k}}{k} M(B \times B \times\{0\} \times\{0\})$,

(D') $\left\langle p_{k}(t), \dot{\bar{x}}_{k}(t)\right\rangle-\lambda_{k} \rho_{F}\left(t, \bar{x}_{k}(t), \dot{\bar{x}}_{k}(t)\right) \geq\left\langle p_{k}(t), v\right\rangle-\lambda_{k} \rho_{F}\left(t, \bar{x}_{k}(t), v\right)-\frac{\lambda_{k}}{k}\left|v-\dot{\bar{x}}_{k}^{e}\right|$ for all $v \in R^{n}$ a.e.,

$\left(\mathrm{E}^{\prime}\right)$

$$
\begin{aligned}
& \lim _{\sigma \rightarrow 0} \operatorname{ess} \inf _{t \in\left[\bar{a}_{k}-\sigma, \bar{a}_{k}\right]}\left(H\left(t, \bar{x}_{k}\left(\bar{a}_{k}\right), p_{k}\left(\bar{a}_{k}\right),\right)-\frac{\lambda_{k}}{k}\left|\bar{u}_{k}(t)\right|\right) \leq \xi_{k} \leq \\
& \leq \lim _{\sigma \rightarrow 0} \operatorname{ess} \sup _{t \in\left[\bar{a}_{k}-\sigma, \bar{a}_{k}\right]}\left(\left\langle p_{k}\left(\bar{a}_{k}\right), \dot{\bar{x}}_{k}(t)\right\rangle-\lambda_{k} \rho_{F}\left(t, \bar{x}_{k}(t), \dot{\bar{x}}_{k}(t)\right)\right.
\end{aligned}
$$

and

$$
\begin{aligned}
& \lim _{\sigma \rightarrow 0} \operatorname{ess} \inf _{t \in\left[\bar{b}_{k}-\sigma, \bar{b}_{k}\right]}\left(H\left(t, \bar{x}_{k}\left(\bar{b}_{k}\right), p_{k}\left(\bar{b}_{k}\right)\right)-\frac{\lambda_{k}}{k}\left|\tilde{u}_{k}(t)\right|\right) \leq \eta_{k} \leq \\
& \leq \lim _{\sigma \rightarrow 0} \operatorname{ess} \sup _{t \in\left[\bar{b}_{k}-\sigma, \bar{b}_{k}\right]}\left(\left\langle p_{k}\left(\bar{b}_{k}\right), \dot{\bar{x}}_{k}(t)\right\rangle-\lambda_{k} \rho_{F}\left(t, \bar{x}_{k}(t), \dot{\bar{x}}_{k}(t)\right),\right.
\end{aligned}
$$

where $\bar{u}_{k}$ and $\tilde{u}_{k}$ are essentially bounded selection of $F\left(\cdot, \bar{x}_{k}\left(\bar{a}_{k}\right)\right)$ and $F\left(\cdot, \bar{x}_{k}\left(\bar{b}_{k}\right)\right)$ respectively which satisfy

$$
\left\langle p_{k}\left(\bar{a}_{k}\right), \bar{u}_{k}(t)\right\rangle=H\left(t, \bar{x}_{k}\left(\bar{a}_{k}\right), p_{k}\left(\bar{a}_{k}\right)\right) \text { a.e. } t \in\left[\bar{a}_{k}-\sigma, \bar{a}_{k}\right]
$$

and

$$
\left\langle p_{k}\left(\bar{b}_{k}\right), \tilde{u}_{k}(t)\right\rangle=H\left(t, \bar{x}_{k}\left(\bar{b}_{k}\right), p_{k}\left(\bar{b}_{k}\right)\right) \text { a.e. } t \in\left[\bar{b}_{k}, \bar{b}_{k}+\sigma\right] .
$$

Note that $\operatorname{esssup} \bar{u}_{k} \leq c_{0}$ and $\operatorname{esssup} \tilde{u}_{k} \leq c_{1}$ for $k$ sufficiently large. By using similar arguments as in part (a), we can assume that $p_{k} \rightarrow p$ uniformly and $\dot{p}_{k} \rightarrow \dot{p}$ weakly in $L^{1}, \lambda_{k} \rightarrow \lambda_{0}, \eta_{k} \rightarrow \eta, \xi_{k} \rightarrow \xi$. By passing to the limits from (A')-(E') we get

(i) $\lambda_{0}+\|p\|_{\infty}=1$.

(ii) $\dot{p}(t) \in \operatorname{co}\left\{\alpha:(\alpha, p(t)) \in N_{\mathrm{Gph} F(t, \cdot)}\left(x_{*}(t), \dot{x}_{*}(t)\right)\right\}$ a.e.

(iii) $\left(-\xi, p\left(a_{*}\right), \eta,-p\left(b_{*}\right)\right) \in N_{C}\left(a_{*}, x_{*}\left(a_{*}\right), b_{*}, x_{*}\left(b_{*}\right)\right)$

(iv) $\langle q(t), \dot{x}(t)\rangle \geq\langle q(t), v\rangle$ for all $v \in F\left(t, x_{*}(t)\right)$ a.e.,

(v) $\xi \in \operatorname{ess}_{t \rightarrow a_{*}} H\left(t, x_{*}\left(a_{*}\right), p\left(a_{*}\right)\right)$ and $\eta \in \operatorname{ess}_{t \rightarrow a_{*}} H\left(t, x_{*}\left(b_{*}\right), p\left(b_{*}\right)\right)$.

We now claim that $\|p\| \neq 0$. Indeed, suppose that $p=0$. Then from the fact $\left(\left[\bar{a}_{k}, \bar{b}_{k}\right], \bar{x}_{k}\right) \notin S_{\epsilon}$ we have either $\left(\bar{a}_{k}, \bar{x}_{k}\left(\bar{a}_{k}\right), \bar{b}_{k}, \bar{x}_{k}\left(\bar{b}_{k}\right)\right) \notin C$ or $\left(\bar{x}_{k}(t), \dot{\bar{x}}_{k}(t)\right) \notin \operatorname{Gph} F(t, \cdot)$. If $\left(\bar{a}_{k}, \bar{x}_{k}\left(\bar{a}_{k}\right), \bar{b}_{k}, \bar{x}_{k}\left(\bar{b}_{k}\right)\right) \notin C$, then (C') implies

$$
\left|\xi_{k}\right|+\left|p_{k}\left(\bar{a}_{k}\right)\right|+\left|\eta_{k}\right|+\left|p_{k}\left(\bar{b}_{k}\right)\right| \geq 2 k \lambda_{k}-\frac{\lambda_{k}}{k}(1+M) .
$$


Hence

$$
\frac{\left|\xi_{k}\right|+\left|p_{k}\left(\bar{a}_{k}\right)\right|+\left|\eta_{k}\right|+\left|p_{k}\left(\bar{b}_{k}\right)\right|}{2 k} \geq \lambda_{k}-\frac{\lambda_{k}}{2 k^{2}}(1+M)
$$

By letting $k \rightarrow \infty$ we get $\lambda_{0}=0$. This contradicts to $\lambda_{0}=1$.

If $\left(\bar{x}_{k}(t), \dot{\bar{x}}_{k}(t)\right) \notin \operatorname{Grap} F(t, \cdot)$ then (D') implies that

$$
p_{k}(t) \in \lambda_{k} \partial_{\dot{x}} \rho_{F}\left(t, \bar{x}_{k}(t), \dot{\bar{x}}_{k}(t)\right)+\frac{\lambda_{k}}{k} B .
$$

By Lemma 2.1, $\left|p_{k}(t)\right| \geq \lambda_{k}-\frac{\lambda_{k}}{k}$. This implies that

$$
\lambda_{k}-\frac{\lambda_{k}}{k} \leq\left\|p_{k}\right\|
$$

By letting $k \rightarrow \infty$ we obtain $\lambda_{0}=0$ which is absurd. Thus it must have $\|p\|=1-\lambda_{0} \neq 0$. By scaling multipliers, we can assume that $\|p\|=1$. Hence we obtain the conclusion of the theorem by putting $\lambda=0$. The proof is complete.

We remark, as pointed out by a referee, that actually the "regularity" (normal semicontinuity) assumption on the preference is not needed in the main Theorem 4.1 if we use the extended limiting normal cone mentioned above for the level set instead of the basic/limiting one. Let us give some corollaries of Theorem 4.1.

When $m=1$, (P) becomes single objective problem. In this case, we have

Corollary 4.2 ([22, Theorem 8.4.1]) Suppose $x_{*}$ is a $W^{1,1}$ local minimizer of $(\mathrm{P})$ and assumptions (H1)-(H4) are satisfied. Then there exist an arc $p \in W^{1,1}$, real numbers $\lambda \geq 0, \xi$ and $\eta$ such that

(i) $\lambda+\|p\|_{\infty}=1$,

(ii) $\dot{p}(t) \in \operatorname{co}\left\{\alpha:(\alpha, p(t)) \in N_{\operatorname{Grph} F(t, \cdot)}\left(x_{*}(t), \dot{x}_{*}(t)\right)\right\}$ a.e.,

(iii) $\left(-\xi, p\left(a_{*}\right), \eta,-p\left(b_{*}\right)\right) \in \lambda \partial g\left(a_{*}, x_{*}\left(a_{*}\right), b_{*}, x_{*}\left(b_{*}\right)\right)+N_{C}\left(a_{*}, x_{*}\left(a_{*}\right), b_{*}, x_{*}\left(b_{*}\right)\right)$,

(iv) $\left\langle p(t), \dot{x}_{*}(t)\right\rangle=H\left(t, x_{*}(t), p(t)\right)$ a.e.t $\in\left[a_{*}, b_{*}\right]$,

(v) $\xi \in \operatorname{ess}_{t \rightarrow a_{*}} H\left(t, x_{*}\left(a_{*}\right), p\left(a_{*}\right)\right)$ and $\eta \in \operatorname{ess}_{t \rightarrow b_{*}} H\left(t, x_{*}\left(b_{*}\right), p\left(b_{*}\right)\right)$.

When $(\mathrm{P})$ is a weak Pareto optimal control problem, we have

Corollary 4.3 Suppose $x_{*}$ is a weak Pareto solution to the multiobjective optimal problem (P) and assumptions (H1)- (H4) are satisfied. Then there exist an arc $p \in W^{1,1}$, real numbers $\lambda \geq 0, \xi, \eta$ a a vector $w \in R_{+}^{m}$ with $\sum_{i=1}^{m} w_{i}=1$ such that

(i) $\lambda+\|p\|_{\infty}=1$,

(ii) $\dot{p}(t) \in \operatorname{co}\left\{\alpha:(\alpha, p(t)) \in N_{\operatorname{Grph} F(t, \cdot)}\left(x_{*}(t), \dot{x}_{*}(t)\right)\right\}$ a.e.,

(iii) $\left(-\xi, p\left(a_{*}\right), \eta,-p\left(b_{*}\right)\right) \in \lambda \partial\left\langle w, g\left(a_{*}, x_{*}\left(a_{*}\right), b_{*}, x_{*}\left(b_{*}\right)\right)\right\rangle+N_{C}\left(a_{*}, x_{*}\left(a_{*}\right), b_{*}, x_{*}\left(b_{*}\right)\right)$,

(iv) $\left\langle p(t), \dot{x}_{*}(t)\right\rangle=H\left(t, x_{*}(t), p(t)\right)$ for a.e. $t \in\left[a_{*}, b_{*}\right]$,

(v) $\xi \in \operatorname{ess}_{t \rightarrow a_{*}} H\left(t, x_{*}\left(a_{*}\right), p\left(a_{*}\right)\right)$ and $\eta \in \operatorname{ess}_{t \rightarrow b_{*}} H\left(t, x_{*}\left(b_{*}\right), p\left(b_{*}\right)\right)$. 
To provide some perspective what we have obtained, in the rest of the paper we give an illustrative example.

Example 4.4 Consider the weak Pareto optimal control problem

Minimize $g(x(b))=\left(x_{1}(b)-x_{2}(b), x_{1}(b)\right)$

over intervals $[0, \mathrm{~b}]$ and $\operatorname{arcs} x=\left(x_{1}, x_{2}\right) \in W^{1,1}\left([0, b], R^{2}\right)$ which satisfy

$$
\left\{\begin{array}{l}
\left(\dot{x}_{1}(t), \dot{x}_{2}(t)\right) \in F(t, x(t)) \\
b \leq 2 \\
\left(x_{1}(0), x_{2}(0)\right)=(0,-2)
\end{array}\right.
$$

where

$$
F(t, x):= \begin{cases}{[-1,1] \times\{1\}} & \text { if } t \leq 1 \\ \{1, t\} \times\{1\} & \text { if } t>1\end{cases}
$$

Evidently, this is problem $(\mathrm{P})$ with the initial time fixed $(a=0)$ and

$$
C=\{0\} \times\{(0,-2)\} \times(-\infty, 2] \times R^{2} .
$$

For each $w=\left(w_{1}, w_{2}\right), w_{1}+w_{2}=1$, we have $\langle w, g(x(b))\rangle=x_{1}(b)-w_{1} x_{2}(b)$. By simple computation, we have

$$
H\left(t,\left(x_{1}, x_{2}\right),\left(p_{1}, p_{2}\right)\right)= \begin{cases}\left|p_{1}\right|+p_{2} & \text { if } t \leq 1 \\ \max \left\{p_{1}+p_{2}, t p_{1}+p_{2}\right\} & \text { if } t>1\end{cases}
$$

We now assume that $([0, b], x)$ is a solution of the problem. By Corollary 4.3, there exist $p$, real numbers $\lambda \geq 0, \eta$ and $w=\left(w_{1}, w_{2}\right) \in R_{+}^{2}, w_{1}+w_{2}=1$ such that assertions $(i)-(v)$ of Corollary 4.3 are satisfied.

Since

$$
\operatorname{Gph} F(t, \cdot)= \begin{cases}R^{2} \times([-1,1] \times\{1\}) & \text { if } t \leq 1 \\ R^{2} \times\{1, t\} \times\{1\} & \text { if } t>1\end{cases}
$$

we get

$$
N_{\mathrm{Gph} F(t, \cdot)}(x(t), \dot{x}(t))= \begin{cases}\{(0,0)\} \times N_{[-1,1] \times\{1\}}(\dot{x}(t)) & \text { if } t \leq 1 \\ \{(0,0)\} \times N_{\{1, t\} \times\{1\}}(\dot{x}(t)) & \text { if } t>1 .\end{cases}
$$

Hence (ii) implies $\dot{p}=(0,0)$. Consequently, $p=\left(p_{1}, p_{2}\right)$, where $p_{1}$ and $p_{2}$ are constants. From (iii) we get

$$
(\eta,-p(b)) \in \lambda\{0\} \times\left\{\left(1,-w_{1}\right)\right\}+N_{(-\infty, 2]}(b) \times\{(0,0\}
$$


This implies that

$$
\eta \in N_{(-\infty, 2]}(b) \text { and } p(b)=\left(p_{1}, p_{2}\right)=\left(-\lambda, \lambda w_{1}\right) .
$$

From (iv) of Corollary 4.3, we obtain the equation

$$
p_{1} \dot{x}_{1}+p_{2} \dot{x}_{2}= \begin{cases}\left|p_{1}\right|+p_{2} & \text { if } t \leq 1 \\ \max \left\{p_{1}+p_{2}, t p_{1}+p_{2}\right\} & \text { if } t>1\end{cases}
$$

for a.e. $t \in[0, b]$. Since $\dot{x}_{2}=1$, we get $x_{2}=t-2$ and

$$
p_{1} \dot{x}_{1}= \begin{cases}\left|p_{1}\right| & \text { if } t \leq 1 \\ \max \left\{p_{1}, t p_{1}\right\} & \text { if } t>1\end{cases}
$$

for a.e. $t \in[0, b]$. Since $p_{1}=-\lambda \leq 0$, we obtain the equation

$$
p_{1} \dot{x}_{1}= \begin{cases}-p_{1} & \text { if } t \leq 1 \\ p_{1} & \text { if } t>1\end{cases}
$$

for a.e. $t \in[0, b]$.

We now consider the following cases.

Case 1. Consider $b<2$. Then we have $\eta=0$ because of $(20)$. By (v) we have $0=$ $H(b, x(b), p(b))$. This implies that $\left|p_{1}\right|+p_{2}=0$ if $0 \leq b \leq 1$ and $p_{1}+p_{2}=0$ if $1<b<2$. Hence if $0 \leq b \leq 1$, then $p=\lambda=0$, which is a contradiction. Thus we must have $1<b<2$ and $0=p_{1}+p_{2}=\lambda\left(w_{1}-1\right)$. It follows that $w_{1}=1$ and $\lambda \neq 0$. From above we obtain

$$
x_{1}= \begin{cases}-t & \text { if } 0 \leq t \leq 1 \\ t-2 & \text { if } 1<t \leq b .\end{cases}
$$

Case 2. Consider $b=2$. From (20) it follows $\eta \geq 0$. By (v) we get

$$
\eta \in H(2, x(2), p(2))=p_{1}+p_{2}=\lambda\left(w_{1}-1\right) .
$$

In this case we also have $p_{1} \neq 0$. So it yields

$$
x_{1}= \begin{cases}-t & \text { if } 0 \leq t \leq 1 \\ t-2 & \text { if } 1<t \leq 2 .\end{cases}
$$

Thus we showed that if $\left(\left[0, b_{*}\right], x_{*}=\left(x_{1 *}, x_{2 *}\right)\right)$ is a solution, then $1<b_{*} \leq 2, x_{2 *}=t-2$,

$$
x_{1 *}= \begin{cases}-t & \text { if } 0 \leq t \leq 1 \\ t-2 & \text { if } 1<t \leq b_{*}\end{cases}
$$




\section{References}

[1] C. Berge, Topological Space, Oliver and Boyd Ltd, 1963.

[2] S. Bellaassali and A. Jourani, Necessary optimality conditions in multiobjective dynamic optimization, SIAM. J. Con. Optim, 42(2004), pp. 2043-2061.

[3] V. Bhaskar, S.K. Gupta and A.K. Ray, Applications of multiobjiective optimization in chemical engineering, Reviews Chem. Eng., 16(2000), pp. 1-54.

[4] J. M. Borwein and Q. J. Zhu, Techniques of Variational Analysis, Springer, 2005.

[5] F. H. Clarke, Optimization and Nonsmooth Analysis, SIAM, Society for Industrial and Applied Mathematics, Philadelphia, 1990.

[6] G. Debreu, Theory of Value, Jonh Wiley and Sons, New York, 1959.

[7] R. Gabasov, F. M. Kirillova and B. Mordukhovich, The discrete maximum principle, Dokl. Akad. Nauk SSSR, 213(1973), pp. 19-22. (Russian; English transl. in Soviet Math. Dokl. 14(1973), pp. 1624-1627).

[8] A. Ioffe, Euler-Lagrange and Hamiltonian formalisms in dynamic optimization, Tran. AMS., 349(1997), pp. 2871-2900.

[9] A. Ioffe and V. M. Tihomirov, Theory of Extremal Problems, North-Holland publishing Company, 1979.

[10] P. D. Loewen and R. T. Rockafellar, Optimal control of unbounded differential inclusions, SIAM J. Control and Optim. 32(1994) pp. 442- 470.

[11] P. D. Loewen and R. T. Rockafellar, Bolza problems with general time constraints, SIAM J. Control Optim. 35(1997), pp. 2050-2069.

[12] B. S. Mordukhovich, Variational Analysis and Generalized Differentiation I, II, Springer, 2006.

[13] B. S. Mordukhovich and N. M. Nam, Variational stability and marginal functions via generalized differentiation, Math. Oper. Res. 30(2005), pp. 800-816.

[14] B. S. Mordukhovich, Optimization and finite difference approximations of nonconvex differential inclusions with free time, in Nonsmooth Analysis and Geometric Method in Deterministic Optimal Control, edited by B.S. Mordukhovick and H. J. Sussmann, Springer, New York (1996), pp. 153-202. 
[15] B. S. Mordukhovich, Discrete approximations and refined Euler-Lagrange conditions for nonconvex differential inclusions, SIAM J. Conttrol Optim., 33 (1995), pp. 882915.

[16] B. S. Mordukhovich, Approximation Methods in Problems of Optimization and Control, Nauka, Moscow 1988.

[17] B. S. Mordukhovich, Nonsmooth Analysis with nonconvex generalized differentials and adjoint mappings, Dokl. Akad. Nauk BSSR 28(1984), pp. 976-979.

[18] B. S. Mordukhovich, Maximum principle in problems of time optimal control with nonsmooth constraints, J. Appl. Math. Mech, 40(1976), pp. 960-969.

[19] R. T. Rockafellar, Hamilton-Jacobi theory and parametric analysis in fully convex problems of optimal control, J. Global Optim. 248(2004), pp. 419-431.

[20] J.D.L. Rowland and R. B. Vinter, Dynamic optimization problems with free time and active state constraints, SIAM J. Control Optim. 31(1993), pp. 677-697.

[21] L. Thibault, On subdifferentials of optimal value functions, SIAM J. Control Optim. 29(1991), pp. 1019-1036.

[22] R. B. Vinter, Optimal Control, Birkhäuser, Boston 2000.

[23] R. B. Vinter and H. Zheng, Necessary conditions for free end-time measurably time dependent optimal control problems with state constraints, Set-Valued Anal. 8(2000), pp. 11-29.

[24] R. B. Vinter and H. Zheng, Necessary conditions for optimal control problems with state constraints, Tran. AMS, 350 (1998), pp. 1181-1204.

[25] R. B. Vinter and H. Zheng, The extended Euler-Lagrange condition for nonconvex variational problems, SIAM J. Control Optim. 35(1997), pp. 56-77.

[26] B. Vroemen and B. De Jager, Multiobjective control: An overview, in Proceedings of the 36th IEEE Conference on Decision and Control, San Diego, CA(1997), pp. 440-445.

[27] Q. J. Zhu, Necessary optimality conditions for nonconvex differential inclusions with endpoint constraints, J. Differential Equations, 124(1996), pp. 186-204, 1996.

[28] Q. J. Zhu, Hamiltonian necessary conditions for a multiobjective optimal control problem with endpoint constraints, SIAM J. Control and Optim, 39(2000), pp. 97112. 